\title{
Untiring Researches for Alternative Resources of Rhizoma Paridis
}

\author{
Xu-Jie Qin ${ }^{1} \cdot$ Wei Ni ${ }^{1} \cdot$ Chang-Xiang Chen ${ }^{1} \cdot$ Hai-Yang Liu ${ }^{1}$ (C) \\ Received: 18 April 2018 / Accepted: 2 July 2018 / Published online: 4 July 2018 \\ (C) The Author(s) 2018
}

\begin{abstract}
Rhizoma Paridis (RP, 重楼), a traditional Chinese medicine, is the rhizoma of Paris polyphylla var. yunnanensis (PPY) or P. polyphylla var. chinensis which are widely used as important raw materials for several Chinese patent drugs. However, the wild resources of these herbs have become less and less due to their slow-growing characteristics and previously excessive excavation. This review covers untiring investigations on alternative resources of RP by our research group over the past decades, including non-medicinal parts of PPY as well as other plants of Liliaceae and Liliflorae families. The arial parts of PPY and the whole plants of Trillium kamtschaticum might be alternative resources for RP based on the fact that they shared the same or similar saponins and bioactivities.
\end{abstract}

Keywords Rhizoma Paridis $\cdot$ Paris polyphylla $\cdot$ Alternative resources $\cdot$ Steroidal saponins $\cdot$ Bioactivities

\section{Introduction}

The genus Paris (Liliaceae) comprises approximately 32 plant species throughout the world and with 26 species found in Southwest China. [1-7]. Among them, the dried rhizoma of Paris polyphylla var. yunnanensis (PPY) and $P$. polyphylla var. chinensis (PPC), both called Rhizoma Paridis (RP) in China, have long been recorded in Chinese Pharmacopoeia as a traditional Chinese medicine to treat furuncle, snakebite, injuries from falls and convulsion, epilepsy, and sore throat [8]. Because of their remarkable medicinal functions, PPY and PPC have been a hot topic within the medicinal chemistry and drug discovery community since the 1970s. Previous studies revealed that PPY and PPC were rich sources of spirostanol (diosgenin and pennogenin) saponins [9-24] responsible for various pharmacological effects, such as cytotoxic and antitumor [13-20], antifungal [21, 22], and haemostatic bioactivities $[23,24]$. The available supplies of PPY and PPC are facing increasing shortage based on the fact that their rhizomes

Hai-Yang Liu

haiyangliu@mail.kib.ac.cn

1 State Key Laboratory of Phytochemistry and Plant Resources in West China, Kunming Institute of Botany, Chinese Academy of Sciences, and Yunnan Key Laboratory of Medicinal Chemistry, Kunming 650201, People's Republic of China can only be harvested until they have grown more than 7 years and the consumption by the pharmaceutical industry of these herbs have increased sharply in recent years. Thus, it is really imperative to search for other saponins or resources that might be substitutes for RP. Over the past 34 years, in order to find valid and alternative resources of RP, our research group have made great effort to phytochemically investigated on the non-medicinal parts of PPY as well as other plants of Liliaceae and Liliflorae families according to their genetic and phylogenetic relationships, which led to the isolation of identical or similar bioactive constituents with those of RP. As a result, a total of 184 saponins and including 120 new ones were obtained and identified, some of which showed interesting bioactive effects as those of RP. This paper mainly describes our untiring researches that can provide active ingredients for alternative resources of RP.

\section{Steroidal Sapogenins and Saponins}

According to the fact that the steroidal saponins are the bioactive constituents of RP, the steroidal sapogenins and saponins of non-medicinal parts of PPY and other Paris, Ypsilandra, Trillium, and Tacca plants have been investigated, which led to the isolation of 17 new steroidal sapogenins and 103 steroidal saponins, along with 64 known analogues. 


\subsection{Non-medicinal Parts of PPY and Other Paris Species (Liliaceae)}

Although the renewable aerial parts of PPY yearly have not been used as medicinal materials, in order to clarify the difference of chemical constituents between medicinal and non-medicinal parts (the stems and leaves) of PPY and to improve the efficiency of resources usage, our systematically phytochemical investigations on the non-medical parts of PPY led to the isolation of 22 new steroidal saponins (Fig. 1; Table 1), named chonglouosides SL-1SL-20 (1-20) [25-27], polyphyllosides III (21) and IV (22)

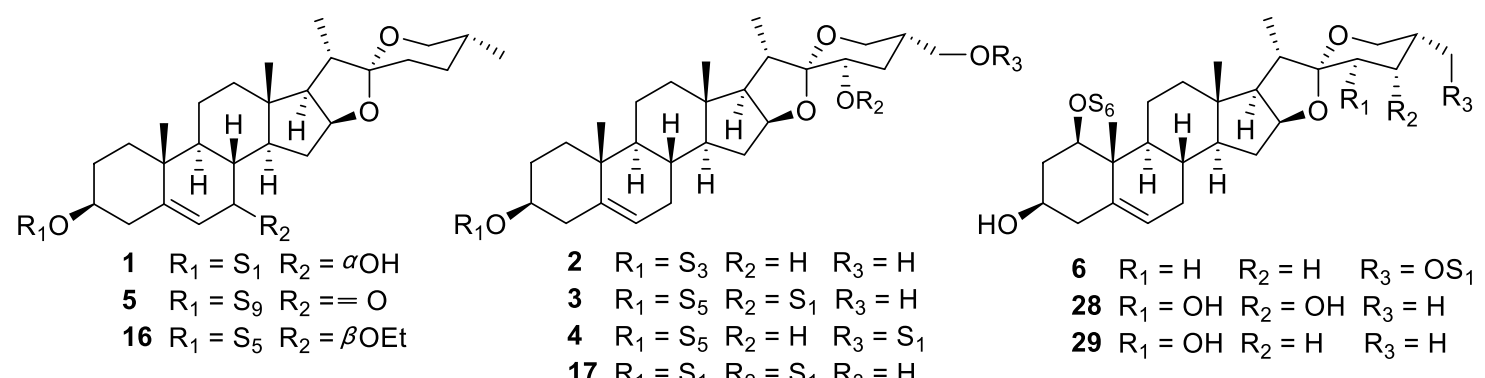

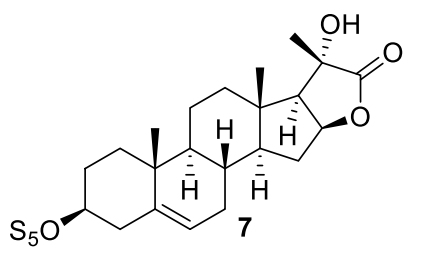<smiles>C=C1C(=O)OC2CC3C4CC=C5CC(OS(=O)(=O)O)CCC5(C)C4CCC3(C)C12</smiles><smiles>[R9]CC1(C)CCC2(OC3CC4C5CC=C6CC([R6])CCC6(C)C5CC([R2])C4(C)C3[C@H]2C)O1</smiles>

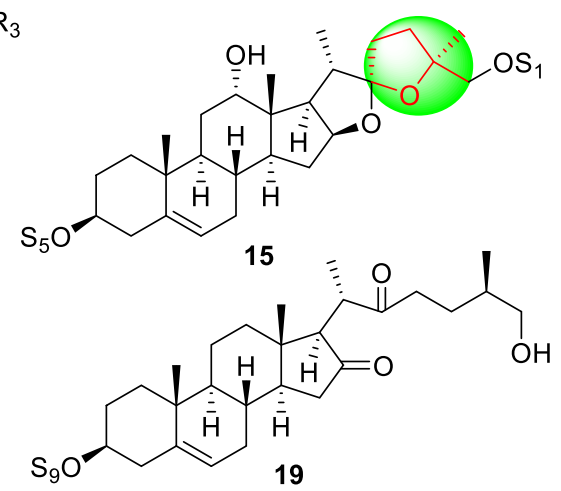

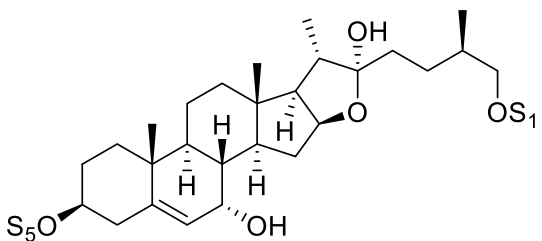

$10 R_{1}=H \quad R_{2}=O H \quad R_{3}=S_{1}$

$\begin{array}{lll}11 \quad R_{1}=S_{1} & R_{2}=H \quad R_{3}=S_{1}\end{array}$

$12 R_{1}=S_{7} R_{2}=H \quad R_{3}=S_{1}$

$13 R_{1}=S_{5} R_{2}=O H \quad R_{3}=S_{1}$

$14 \mathrm{R}_{1}=\mathrm{S}_{9} \mathrm{R}_{2}=\mathrm{OH} \mathrm{R}_{3}=\mathrm{S}_{1}$

$28 \mathrm{R}_{1}=\mathrm{OH} \mathrm{R}_{2}=\mathrm{OH} \mathrm{R}_{3}=\mathrm{H}$

$29 \mathrm{R}_{1}=\mathrm{OH} \mathrm{R}_{2}=\mathrm{H} \quad \mathrm{R}_{3}=\mathrm{H}$

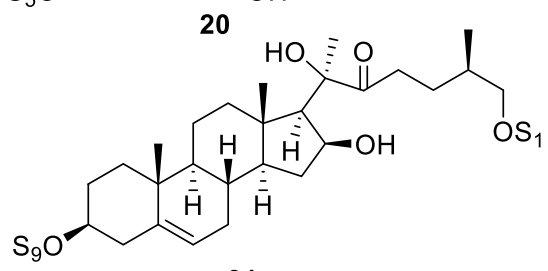

31

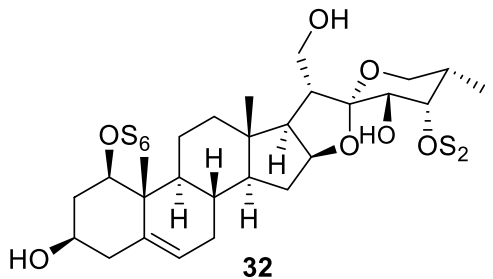

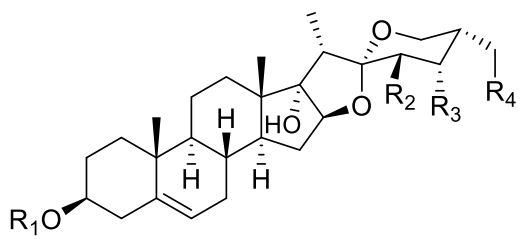

$21 \quad R_{1}=S_{5} R_{2}=H \quad R_{3}=H \quad R_{4}=O H$

$22 \mathrm{R}_{1}=\mathrm{S}_{5} \quad \mathrm{R}_{2}=\mathrm{OH} \quad \mathrm{R}_{3}=\mathrm{H} \quad \mathrm{R}_{4}=\mathrm{OH}$

$23 \quad R_{1}=H_{2} \quad R_{2}=H \quad R_{3}=H \quad R_{4}=O H$

$24 \quad R_{1}=H_{2} \quad R_{2}=O H \quad R_{3}=H \quad R_{4}=O H$

$\begin{array}{llll}25 & R_{1}=S_{7} & R_{2}=H & R_{3}=H \\ R_{4}=H\end{array}$

$26 R_{1}=S_{7} R_{2}=H \quad R_{3}=O H \quad R_{4}=H$

$27 \quad R_{1}=S_{8} \quad R_{2}=H \quad R_{3}=O H \quad R_{4}=H$

$30 R_{1}=S_{9} R_{2}=H S_{2} R_{3}=H \quad R_{4}=O S_{1}$
$\mathrm{S}_{2}=\beta$-D-Gal-
$\mathrm{S}_{3}=\alpha$-L-Rha- $(1 \rightarrow 4)-\beta$-D-GlC- $\quad \mathrm{S}_{4}=\beta$-D-GlC- $(1 \rightarrow 6)-\beta$-D-GlC-
$\mathrm{S}_{5}=\alpha-$ L-Rha- $(1 \rightarrow 2)-[\alpha-$ L-Rha- $(1 \rightarrow 4)]-\beta$-D-GlC-
$\mathrm{S}_{6}=\alpha$-L-Rha- $(1 \rightarrow 2)-[\beta$-D-Xyl-( $(1 \rightarrow 3)]-\beta$-D-GIC-
$\mathrm{S}_{7}=\alpha-\mathrm{L}-\mathrm{Rha}-(1 \rightarrow 2)-[\beta$-D-GlC- $(1 \rightarrow 3)]-\beta$-D-GlC-
$\mathrm{S}_{8}=\alpha$-L-Rha- $(1 \rightarrow 2)-[\alpha-\mathrm{L}-\mathrm{Ara}-(1 \rightarrow 4)]-\beta$-D-GlC-
$\mathrm{S}_{9}=\alpha$-L-Rha- $(1 \rightarrow 2)-[\alpha-\mathrm{L}-\mathrm{Rha} a-(1 \rightarrow 4)-\mathrm{L}-\mathrm{Rha}-(1 \rightarrow 4)]-\beta$-D-GlC-

Fig. 1 New steroidal sapogenins and saponins from non-medicinal parts of PPY and other Paris species 
Table 1 New steroidal sapogenins and saponins from non-medicinal parts of PPY and other Paris species

\begin{tabular}{|c|c|c|c|c|}
\hline Nos. & Names & Species & Parts & References \\
\hline 1 & Chonglouoside SL-1 & PPY & Stems and leaves & {$[25]$} \\
\hline 2 & Chonglouoside SL-2 & PPY & Stems and leaves & {$[25]$} \\
\hline 3 & Chonglouoside SL-3 & PPY & Stems and leaves & {$[25]$} \\
\hline 4 & Chonglouoside SL-4 & PPY & Stems and leaves & {$[25]$} \\
\hline 5 & Chonglouoside SL-5 & PPY & Stems and leaves & {$[25]$} \\
\hline 6 & Chonglouoside SL-6 & PPY & Stems and leaves & {$[25]$} \\
\hline 7 & Chonglouoside SL-7 & PPY & Stems and leaves & {$[26]$} \\
\hline 8 & Chonglouoside SL-8 & PPY & Stems and leaves & {$[26]$} \\
\hline 9 & Chonglouoside SL-9 & PPY & Stems and leaves & {$[27]$} \\
\hline 10 & Chonglouoside SL-10 & PPY & Stems and leaves & {$[27]$} \\
\hline 11 & Chonglouoside SL-11 & PPY & Stems and leaves & {$[27]$} \\
\hline 12 & Chonglouoside SL-12 & PPY & Stems and leaves & {$[27]$} \\
\hline 13 & Chonglouoside SL-13 & PPY & Stems and leaves & {$[27]$} \\
\hline 14 & Chonglouoside SL-14 & PPY & Stems and leaves & {$[27]$} \\
\hline 15 & Chonglouoside SL-15 & PPY & Stems and leaves & {$[27]$} \\
\hline 16 & Chonglouoside SL-16 & PPY & Stems and leaves & {$[27]$} \\
\hline 17 & Chonglouoside SL-17 & PPY & Stems and leaves & {$[27]$} \\
\hline 18 & Chonglouoside SL-18 & PPY & Stems and leaves & {$[27]$} \\
\hline 19 & Chonglouoside SL-19 & PPY & Stems and leaves & {$[27]$} \\
\hline 20 & Chonglouoside SL-20 & PPY & Stems and leaves & {$[27]$} \\
\hline 21 & Polyphylloside III & PPY & Aerial parts & {$[28]$} \\
\hline 22 & Polyphylloside IV & PPY & Aerial parts & {$[28]$} \\
\hline 23 & 27-Hydroxyl-pennogenin & PPY & Aerial parts & [29] \\
\hline 24 & $27,23 \beta$-Dihydroxyl-pennogenin & PPY & Aerial parts & [29] \\
\hline 25 & $\begin{array}{l}\text { Pennogenin-3-O- } \beta \text {-D-glucopyranosyl- }(1 \rightarrow 3) \text { - } \\
\quad[\alpha \text {-L-rhamnopyranosyl }(1 \rightarrow 2)] \text { - } \beta \text {-D-glucopyranoside }\end{array}$ & P. axialis & Rhizomes & {$[30]$} \\
\hline 26 & $\begin{array}{l}\text { 24 } \alpha \text {-Hydroxyl-pennogenin-3-O- } \beta \text {-D-glucopyranosyl- } \\
\quad(1 \rightarrow 3) \text {-[ } \alpha \text {-L-rhamnopyranosyl }(1 \rightarrow 2)]-\beta \text {-D-glucopyranoside }\end{array}$ & P. axialis & Rhizomes & {$[30]$} \\
\hline 27 & $\begin{array}{l}\text { 24 } \alpha \text {-Hydroxyl-pennogenin-3- } O \text { - } \alpha \text {-L-rhamnopyranosyl- } \\
(1 \rightarrow 2) \text {-[ } \alpha \text {-L-arabinofuranosyl }(1 \rightarrow 4)]-\beta \text {-D-glucopyranoside }\end{array}$ & P. axialis & Rhizomes & {$[31]$} \\
\hline 28 & Parisverticoside A & P. verticillata & Aerial parts & {$[32]$} \\
\hline 29 & Parisverticoside B & P. verticillata & Aerial parts & {$[32]$} \\
\hline 30 & Parisverticoside $\mathrm{C}$ & P. verticillata & Aerial parts & {$[32]$} \\
\hline 31 & Parisverticoside D & P. verticillata & Aerial parts & {$[32]$} \\
\hline 32 & Paristenoside A & P. polyphylla var. stenophylla & Rhizomes & {$[33]$} \\
\hline 33 & Paristenoside B & P. polyphylla var. stenophylla & Rhizomes & [33] \\
\hline
\end{tabular}

[28], as well as two new steroidal sapogenins, named 27-hydroxylpennogenin (23) and 27,23ß-dihydroxylpennogenin (24) [29]. In addition, three new pennogenin saponins (25-27) [30, 31], three new spirostanol saponins (28-30) and one new cholestane saponin (31) [32], and two new highly oxygenated spirostanol saponins (32 and 33) [33] were isolated from $P$. axialis (rhizomes), $P$. verticillata (aerial parts), and P. polyphylla var. stenophylla (rhizomes), respectively (Fig. 1). It was worth noting that saponins 7 and $\mathbf{8}$ were $\mathrm{C}_{22}$-steroidal lactone saponins which were isolated from genus Paris for the first time, while 9-
15 were rare nuatigenin saponins with a furan ring that firstly obtained from species of Liliaceae family.

\subsection{Ypsilandra Species (Liliaceae)}

Ypsilandra (Liliaceae), a small genus including only five species, is widely distributed in Southwest China and Myanmar [34]. We speculate that Ypsilandra species should produce similar steroidal derivatives as those of Paris due to their genetic and phylogenetic relationships. Although $Y$. thibetica has been used as a folk medicine for treating uterine 
bleeding and traumatic hemorrhage [35, 36], the chemical constituents of Ypsilandra species have not been studied before our investigations. A total of two new sapogenins and 38 saponins (Fig. 2; Table 2) have been reported from the whole plants of $Y$. thibetica, Y. parviflora, and $Y$. yunnanensis up to 2017 by our research group, namely, isoypsilandrogenin (34), isoypsilandrosides A (35) and B (36), ypsilandrosides A (37) and B (38) [37], ypsilandrosides C-G (3943) [38], ypsilandrosides H-L (44-48) [39], ypsilandrosides M-O (49-51) [40], ypsiparosides A-G (52-58) [41], ypsilanogenin (59), ypsilanogenin 3-O- $\beta$-D-glucopyranoside (60), 4'-acetylypsilanogenin 3-O- $\beta$-D-glucopyranoside (61) [42], ypsilandrosides P-R (62-64) [43], ypsilandrosides S (65) and T (66) [44], ypsiyunnosides A-E (67-71) [45], and ypsilactosides A (71) and B (72) [46]. These new saponins were usually the oxygenated derivatives at C-6, C-7, C-11, and $\mathrm{C}-12$ of those known analogues and some of these isolates had unpredicted aglycones. To be more specific, saponins 44 and $\mathbf{4 5}$ represented the first example with a novel $5(6 \rightarrow 7)$ abeo-steroidal aglycone, whereas 59-61 were unusual 23-spirocholestane derivatives and 67 possessed a rare 6/6/6/5/5 fused-rings cholestanol skeleton.

\subsection{Trillium Species (Liliaceae)}

The Trillium genus consists of approximately 49 species throughout the world. However, only three species, $T$. kamtschaticum, T. tschonoskii, and T. govanianum, are found in Hubei, Sichuan, Yunnan, and Xizang Provinces of China. The rhizomes of T. kamtschaticum, called "Toudingyikezhu" in Chinese, have been traditionally use by Chinese minorities (Tujia and Miao people) for the treatment of traumatic hemorrhage [47, 48]. In addition, some pennogenin saponins have been reported from Trillium species $[49,50]$ and the crude extract of the whole plants of T. kamtschaticum displayed significant induced-platelet aggregation activity at a concentration of $1.5 \mathrm{mg} / \mathrm{mL}$ as revealed by our initiatory test. All these information strongly inspired us to investigated the hemostatic constituents of the whole plats of T. kamtschaticum, resulting in the isolation of 18 new steroidal saponins (Fig. 3; Table 3), named trillikamtosides A-R (74-91) [51, 52]. Interestingly, some of them were determined to have rare aglycone moieties. For instance, the aglycones of 73-75 had unique $3 \beta, 17 \alpha$-dihydroxyspirostanes featuring a double
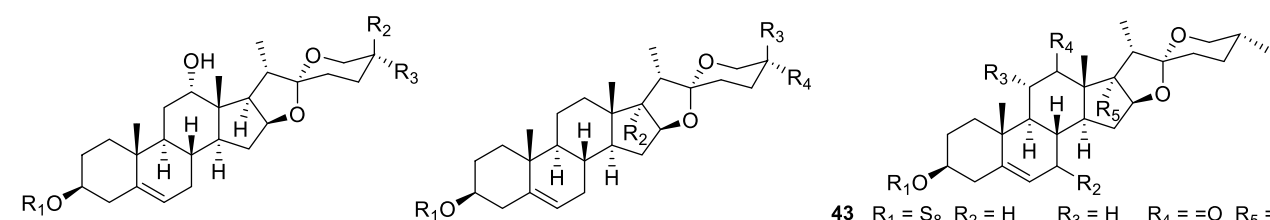

$\begin{array}{llllll}34 & \mathrm{R}_{1}=\mathrm{H} & \mathrm{R}_{2}=\mathrm{H} & \mathrm{R}_{3}=\mathrm{CH}_{2} \mathrm{OH} & 39 \quad \mathrm{R}_{1}=\mathrm{S}_{3} \mathrm{R}_{2}=\mathrm{OH} \mathrm{R}_{3}=\mathrm{H} \mathrm{R}_{4}=\mathrm{CH}_{2} \mathrm{OH} \\ 35 & \mathrm{R}_{1}=\mathrm{S}_{1} & \mathrm{R}_{2}=\mathrm{H} & \mathrm{R}_{3}=\mathrm{CH}_{2} \mathrm{OH} & \mathbf{4 0} \mathrm{R}_{1}=\mathrm{S}_{6} \mathrm{R}_{2}=\mathrm{OH} \mathrm{R}_{3}=\mathrm{H} \mathrm{R} \mathrm{R}_{4}=\mathrm{CH}_{2} \mathrm{OH} \\ 36 & \mathrm{R}_{1}=\mathrm{S}_{3} \mathrm{R}_{2}=\mathrm{H} & \mathrm{R}_{3}=\mathrm{CH}_{2} \mathrm{OH} \quad \mathbf{4 2} \mathrm{R}_{1}=\mathrm{S}_{6} \mathrm{R}_{2}=\mathrm{H} \quad \mathrm{R}_{3}=\mathrm{H} \mathrm{R}_{4}=\mathrm{CH}_{2} \mathrm{OH}\end{array}$

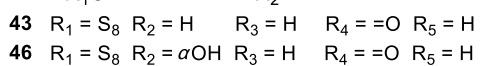

$47 \mathrm{R}_{1}=\mathrm{S}_{8} \quad \mathrm{R}_{2}=\alpha \mathrm{OH} \mathrm{R}_{3}=\mathrm{OH} \mathrm{R}_{4}==\mathrm{O} \mathrm{R}_{5}=\mathrm{H}$

$37 \mathrm{R}_{1}=\mathrm{S}_{1} \mathrm{R}_{2}=\mathrm{CH}_{2} \mathrm{OH} \mathrm{R}_{3}=\mathrm{H}$

$38 \mathrm{R}_{1}=\mathrm{S}_{3} \mathrm{R}_{2}=\mathrm{CH}_{2} \mathrm{OH} \mathrm{R}_{3}=\mathrm{H}$

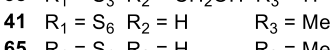

$\begin{array}{llll}50 \mathrm{R}_{1}=\mathrm{S}_{8} \mathrm{R}_{2}=\beta \mathrm{OH} \mathrm{R}_{3}=\mathrm{H} & \mathrm{R}_{4}=\mathrm{H} & \mathrm{R}_{5}=\mathrm{H}\end{array}$

$\begin{array}{llll}51 \quad \mathrm{R}_{1}=\mathrm{S}_{8} & \mathrm{R}_{2}=\alpha \mathrm{OH} \quad \mathrm{R}_{3}=\mathrm{H} & \mathrm{R}_{4}=\mathrm{H} & \mathrm{R}_{5}=\mathrm{OH}\end{array}$

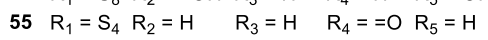

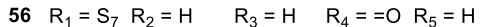

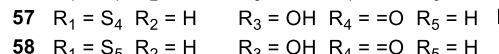

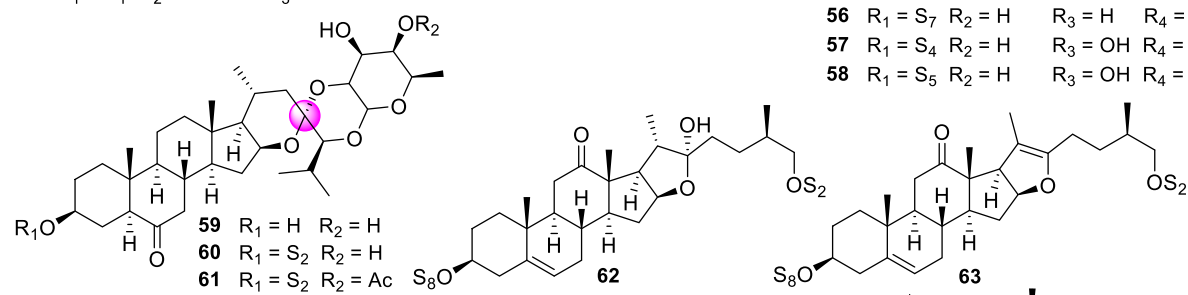

$\overbrace{\mathrm{OS}_{2}}$

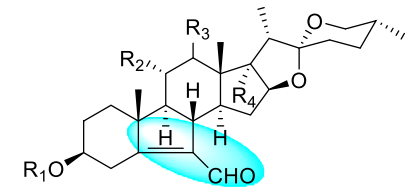

$44 \quad \mathrm{R}_{1}=\mathrm{S}_{8} \quad \mathrm{R}_{2}=\mathrm{H} \quad \mathrm{R}_{3}=\mathrm{H} \quad \mathrm{R}_{4}=\mathrm{OH}$

$45 R_{1}=S_{8} R_{2}=O H \quad R_{3}==O R_{4}=H$
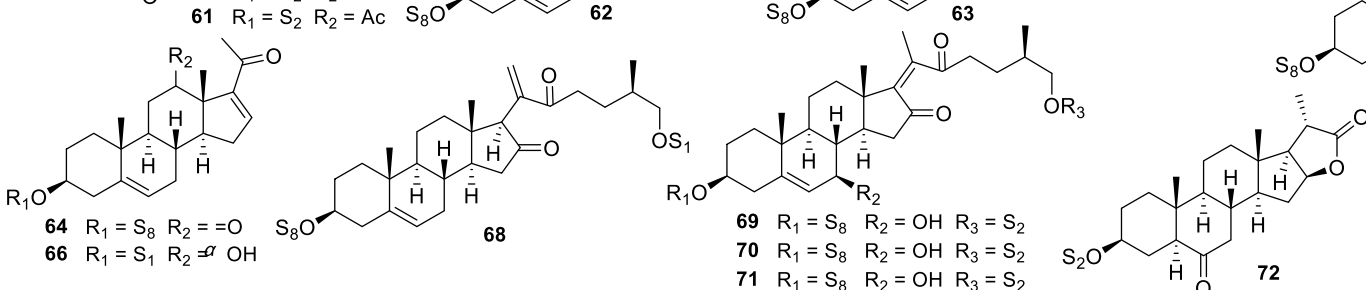

$\begin{array}{lll}48 & \mathrm{R}_{1}=\mathrm{S}_{7} \mathrm{R}_{2}=\mathrm{H} & \mathrm{R}_{3}=\mathrm{CH}_{2} \mathrm{OH} \\ 52 & \mathrm{R}_{1}=\mathrm{S}_{1} \mathrm{R}_{2}=\mathrm{H} & \mathrm{R}_{3}=\mathrm{CH}_{2} \mathrm{OH}\end{array}$ $52 \mathrm{R}_{1}=\mathrm{S}_{1} \mathrm{R}_{2}=\mathrm{H} \quad \mathrm{R}_{3}=\mathrm{CH}_{2} \mathrm{OH}$ $53 \quad \mathrm{R}_{1}=\mathrm{S}_{2} \mathrm{R}_{2}=\mathrm{H} \quad \mathrm{R}_{3}=\mathrm{CH}_{2} \mathrm{OH}$ $54 \mathrm{R}_{1}=\mathrm{S}_{2} \mathrm{R}_{2}=\mathrm{CH}_{2} \mathrm{OH} \mathrm{R}_{3}=\mathrm{H}$

$\begin{array}{ll}64 & \mathrm{R}_{1}=\mathrm{S}_{8} \mathrm{R}_{2}==\mathrm{O} \\ 66 & \mathrm{R}_{1}=\mathrm{S}_{1} \mathrm{R}_{2}=\alpha \mathrm{OH}\end{array}$ 68

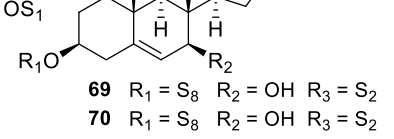
$\begin{array}{lll}70 & R_{1}=S_{8} & R_{2}=O H R_{3}=S_{2} \\ 71 & R_{1}=S_{8} & R_{2}=O H R_{3}=S_{2}\end{array}$

$\mathrm{S}_{1}=\beta$-D-Api- $\mathrm{S}_{2}=\beta$-D-GlC- $\quad \mathrm{S}_{3}=\alpha$-L-Rha- $(1 \rightarrow 4)-\beta$-D-Api- $\quad \mathrm{S}_{4}=\alpha$-L-Rha- $(1 \rightarrow 2)-\beta$-D-GlC-

$\mathrm{S}_{5}=\alpha$-L-Rha- $(1 \rightarrow 2)-[\alpha$-L-Rha- $(1 \rightarrow 4)]-\beta$-D-GlC- $\quad \mathrm{S}_{6}=\alpha$-L-Rha- $(1 \rightarrow 2)-[\alpha-$ L-Rha- $(1 \rightarrow 5)]-\beta$-D-Api-

$\mathrm{S}_{7}=\alpha$-L-Rha- $(1 \rightarrow 4)$-[ $\alpha$-L-Rha- $\left.(1 \rightarrow 4)\right]-\beta$-D-GlC- $\quad \mathrm{S}_{8}=\alpha$-L-Rha- $(1 \rightarrow 2)-[\alpha-$ L-Rha- $(1 \rightarrow 4)-$ L-Rha- $(1 \rightarrow 4)]-\beta-\mathrm{D}-\mathrm{GlC}-$

Fig. 2 New steroidal sapogenins and saponins from Ypsilandra species 
Table 2 New steroidal sapogenins and saponins from Ypsilandra species (Liliaceae)

\begin{tabular}{|c|c|c|c|c|}
\hline Nos. & Names & Species & Parts & References \\
\hline 34 & Isoypsilandrogenin & Y. thibetica & Whole plants & {$[37]$} \\
\hline 35 & Isoypsilandroside A & Y. thibetica & Whole plants & {$[37]$} \\
\hline 36 & Isoypsilandroside B & Y. thibetica & Whole plants & {$[37]$} \\
\hline 37 & Ypsilandroside A & Y. thibetica & Whole plants & [37] \\
\hline 38 & Ypsilandroside B & Y. thibetica & Whole plants & {$[37]$} \\
\hline 39 & Ypsilandroside C & Y. thibetica & Whole plants & [38] \\
\hline 40 & Ypsilandroside D & Y. thibetica & Whole plants & {$[38]$} \\
\hline 41 & Ypsilandroside E & Y. thibetica & Whole plants & {$[38]$} \\
\hline 42 & Ypsilandroside F & Y. thibetica & Whole plants & {$[38]$} \\
\hline 43 & Ypsilandroside G & Y. thibetica & Whole plants & {$[38]$} \\
\hline 44 & Ypsilandroside $\mathrm{H}$ & Y. thibetica & Whole plants & [39] \\
\hline 45 & Ypsilandroside I & Y. thibetica & Whole plants & [39] \\
\hline 46 & Ypsilandroside $\mathrm{J}$ & Y. thibetica & Whole plants & [39] \\
\hline 47 & Ypsilandroside $\mathrm{K}$ & Y. thibetica & Whole plants & [39] \\
\hline 48 & Ypsilandroside L & Y. thibetica & Whole plants & [39] \\
\hline 49 & Ypsilandroside $\mathrm{M}$ & Y. thibetica & Whole plants & {$[40]$} \\
\hline 50 & Ypsilandroside $\mathrm{N}$ & Y. thibetica & Whole plants & {$[40]$} \\
\hline 51 & Ypsilandroside $\mathrm{O}$ & Y. thibetica & Whole plants & {$[40]$} \\
\hline 52 & Ypsiparoside A & Y. parviflora & Whole plants & {$[40]$} \\
\hline 53 & Ypsiparoside B & Y. parviflora & Whole plants & [41] \\
\hline 54 & Ypsiparoside C & Y. parviflora & Whole plants & {$[41]$} \\
\hline 55 & Ypsiparoside D & Y. parviflora & Whole plants & {$[41]$} \\
\hline 56 & Ypsiparoside E & Y. parviflora & Whole plants & {$[41]$} \\
\hline 57 & Ypsiparoside F & Y. parviflora & Whole plants & {$[41]$} \\
\hline 58 & Ypsiparoside G & Y. parviflora & Whole plants & {$[41]$} \\
\hline 59 & Ypsilanogenin & Y. thibetica & Whole plants & {$[42]$} \\
\hline 60 & Ypsilanogenin 3-O- $\beta$-D-glucopyranoside & Y. thibetica & Whole plants & {$[42]$} \\
\hline 61 & 4'-Acetylypsilanogenin 3-O- $\beta$-D-glucopyranoside & Y. thibetica & Whole plants & {$[42]$} \\
\hline 62 & Ypsilandroside $\mathrm{P}$ & Y. thibetica & Whole plants & {$[43]$} \\
\hline 63 & Ypsilandroside Q & Y. thibetica & Whole plants & {$[43]$} \\
\hline 64 & Ypsilandroside $\mathrm{R}$ & Y. thibetica & Whole plants & {$[43]$} \\
\hline 65 & Ypsilandroside S & Y. thibetica & Whole plants & {$[44]$} \\
\hline 66 & Ypsilandroside $\mathrm{T}$ & Y. thibetica & Whole plants & {$[44]$} \\
\hline 67 & Ysiyunnoside A & Y. yunnanensis & Whole plants & {$[45]$} \\
\hline 68 & Ysiyunnoside B & Y. yunnanensis & Whole plants & [45] \\
\hline 69 & Ysiyunnoside $\mathrm{C}$ & Y. yunnanensis & Whole plants & {$[45]$} \\
\hline 70 & Ysiyunnoside D & Y. yunnanensis & Whole plants & {$[45]$} \\
\hline 71 & Ysiyunnoside E & Y. yunnanensis & Whole plants & {$[45]$} \\
\hline 72 & Ypsilactoside A & Y. thibetica & Whole plants & {$[46]$} \\
\hline 73 & Ypsilactoside B & Y. thibetica & Whole plants & [46] \\
\hline
\end{tabular}

bond between $\mathrm{C}-4$ and $\mathrm{C}-5,76$ and 77 represented a rare class of spirostanol saponins which possess a 5(6-7) abeosteroidal aglycone, and $\mathbf{8 3}$ possessed a rare aglycone with a 16-oxaandrost-5-en-3-ol-17-one moiety. Moreover, saponins 84 and 86 were schizolytic derivatives of those furanstanols and 89-91 were new trillenogenin saponins being only found in Trillium plants. The relevant researches of the other Trillium species are going on in our laboratory. 


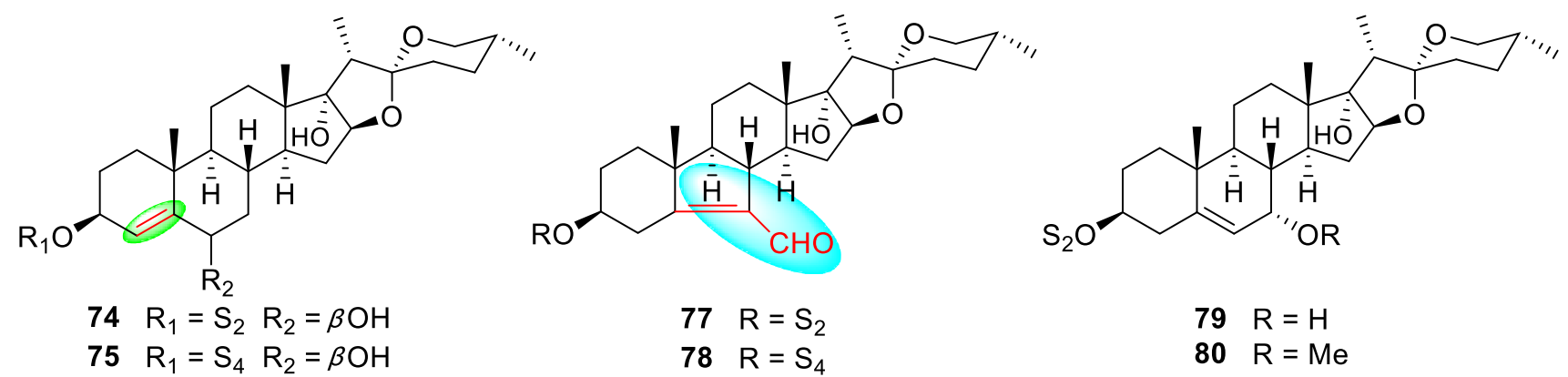

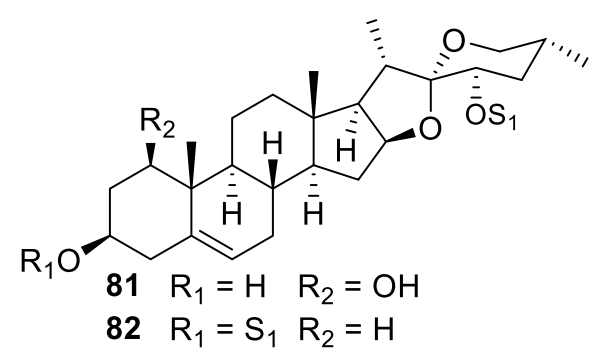

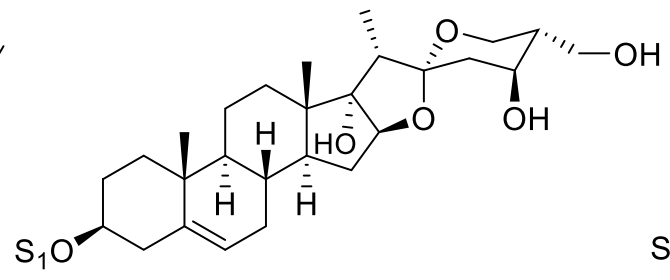

$83 \mathrm{R}_{1}=\mathrm{H} \quad \mathrm{R}_{2}=\mathrm{OH}$

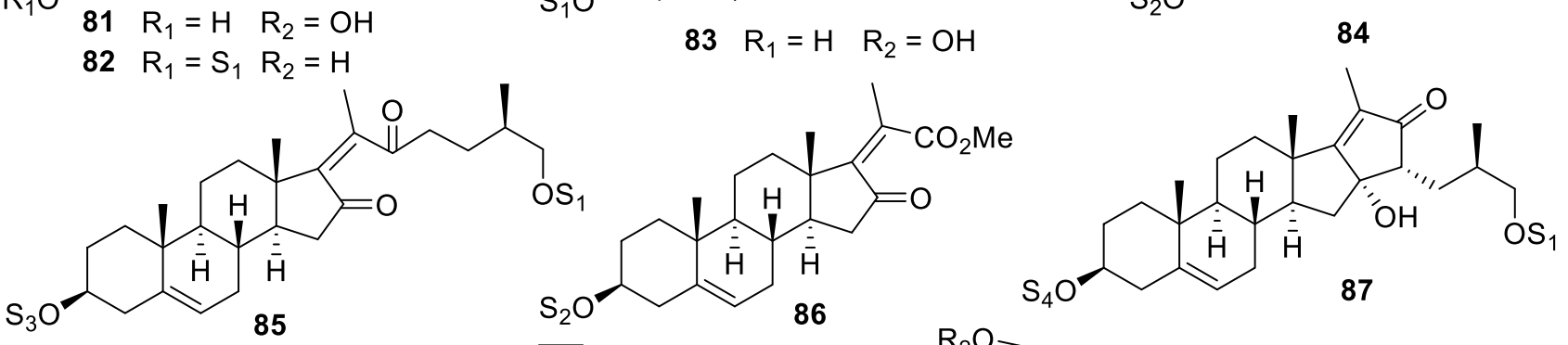
85 86

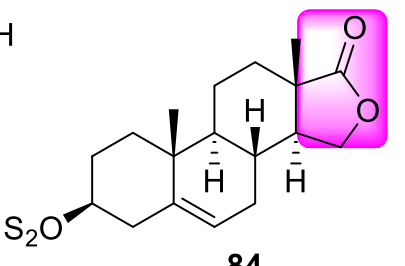<smiles>Cc1c(CCC(C)CO)ccc2c1[C@]1(C)CC[C@H]3C(CC=C4C[C@@H](OS(C)(=O)=O)CC[C@]43C)[C@@H]1C2</smiles>

$\mathrm{S}_{1}=\beta$-D-GlC- $\quad \mathrm{S}_{2}=\alpha$-L-Rha- $(1 \rightarrow 2)-\beta$-D-GlC-

$\mathrm{S}_{3}=\alpha-$ L-Rha- $(1 \rightarrow 4)-\beta$-D-GlC-

$\mathrm{S}_{4}=\alpha-\mathrm{L}-\mathrm{Rha}-(1 \rightarrow 2)-[\alpha-\mathrm{L}-\mathrm{Rha}-(1 \rightarrow 4)]-\beta$-D-GlC-

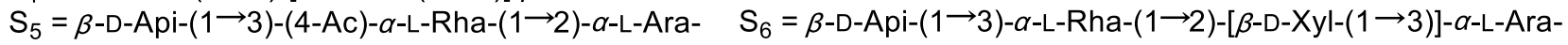

Fig. 3 New steroidal saponins from Trillium species

\subsection{Tacca Species (Taccaceae)}

Compared with the genera of Liliaceae family, the Tacca plants are very limited. In order to discuss/explore whether the Tacca species possess the same steroidal constituents as that of RP, our group investigated the phytochemicals of two Tacca species (T. plantaginea and T. subflabellata). The results led to the structural characterization of eight new spirostane saponins, named taccaosides E-L (92-99) [53], taccaoside C (100) [54], taccasubosides B (103) and $C$ (104) [55], three furostanol saponins, named taccaoside D (101) [54], taccaosides A (106) and B (107) [56], a new $\mathrm{C}_{21}$ steroidal saponin, taccasuboside D (105) [55], and 13 new withanolides, named taccasuboside A (102) [55], plantagiolides A-E (108-112) [57], plantagiolide F (113)
[58], plantagiolides $\mathrm{K}-\mathrm{N}$ (114-117) [59], and taccalonolides W-Y (118-120) [60] (Fig. 4; Table 4). Although withanolides 108-117 and taccalonolides 118-120 were also steroidal derivatives with 28 carbons, they may be the taxonomic markers of Tacca species.

\subsection{Known Sapogenin and Saponins Obtained from the Non-medicinal Parts of PPY and Other Paris, Ypsilandra, Trillium, and Tacca Plants}

Apart from the above mentioned new saponins, 1 known sapogenin and 63 known saponins were also identified from the aforementioned species (Fig. 5; Table 5). 
Table 3 New steroidal saponins from the whole plants of $T$. kamtschaticum

\begin{tabular}{llll}
\hline No. & Name & Species & References \\
\hline $\mathbf{7 4}$ & Trillikamtoside A & T. kamtschaticum & {$[51]$} \\
$\mathbf{7 5}$ & Trillikamtoside B & T. kamtschaticum & {$[51]$} \\
$\mathbf{7 6}$ & Trillikamtoside C & T. kamtschaticum & {$[51]$} \\
$\mathbf{7 7}$ & Trillikamtoside D & T. kamtschaticum & {$[51]$} \\
$\mathbf{7 8}$ & Trillikamtoside E & T. kamtschaticum & {$[51]$} \\
$\mathbf{7 9}$ & Trillikamtoside F & T. kamtschaticum & {$[51]$} \\
$\mathbf{8 0}$ & Trillikamtoside G & T. kamtschaticum & {$[51]$} \\
$\mathbf{8 1}$ & Trillikamtoside H & T. kamtschaticum & {$[51]$} \\
$\mathbf{8 2}$ & Trillikamtoside I & T. kamtschaticum & {$[51]$} \\
$\mathbf{8 3}$ & Trillikamtoside J & T. kamtschaticum & {$[51]$} \\
$\mathbf{8 4}$ & Trillikamtoside K & T. kamtschaticum & {$[52]$} \\
$\mathbf{8 5}$ & Trillikamtoside L & T. kamtschaticum & {$[52]$} \\
$\mathbf{8 6}$ & Trillikamtoside M & T. kamtschaticum & {$[52]$} \\
$\mathbf{8 7}$ & Trillikamtoside N & T. kamtschaticum & {$[52]$} \\
$\mathbf{8 8}$ & Trillikamtoside O & T. kamtschaticum & {$[52]$} \\
$\mathbf{8 9}$ & Trillikamtoside P & T. kamtschaticum & {$[52]$} \\
$\mathbf{9 0}$ & Trillikamtoside Q & T. kamtschaticum & {$[52]$} \\
$\mathbf{9 1}$ & Trillikamtoside R & T. kamtschaticum & {$[52]$} \\
\hline & & &
\end{tabular}

Compared with those new isolates, these known compounds usually shared the aglycones with lower oxidation degrees.

\section{Bioactivities}

Based on the fact that RP is traditionally used as hemostatic, antimicrobial, and antitumor agents, the hemostatic, antimicrobial, and cytotoxic activities of obtained compounds were evaluated to initially confirm that whether the plants could be alternative resources of RP. Our studies revealed that most of the bioactive compounds were spirostanol saponins with only one sugar chain at $\mathrm{OH}-3$.

\subsection{Hemostatic Effect}

Both the total steroidal saponin moieties and purified saponins of PPY and T. kamtschaticum exhibited hemostatic effects. The $70 \% \mathrm{EtOH}$ eluted fraction of T. kamtschaticum crude extract obtained from a macroporous resin column showed $76 \%$ maximal platelet aggregation rate at a concentration of $1.5 \mathrm{mg} / \mathrm{mL}$ [51]. Subsequently, three
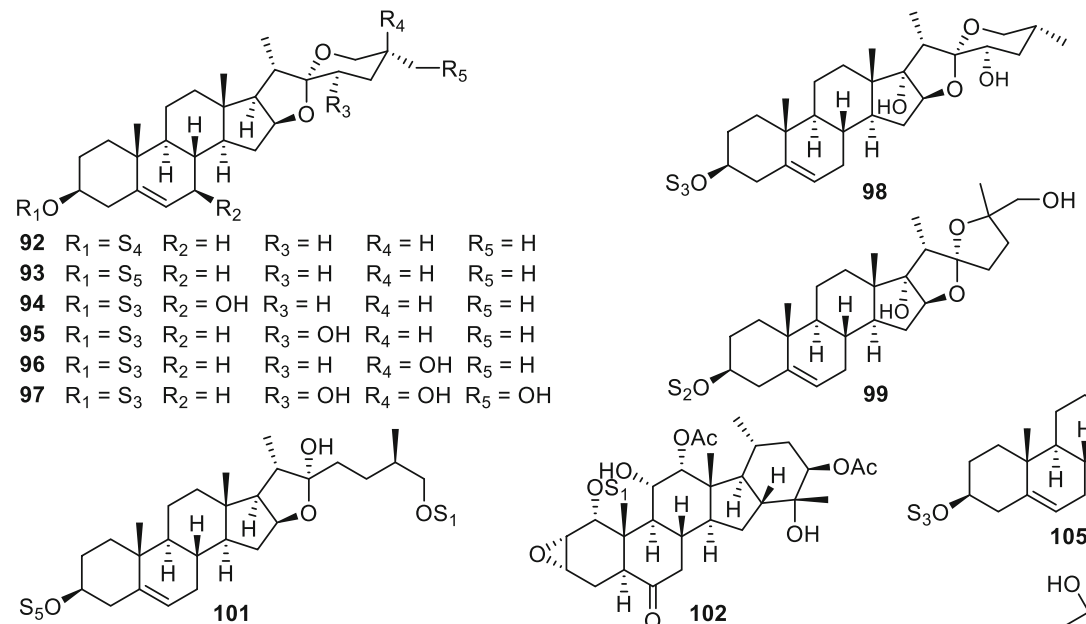

(n)

$108 R_{1}=A c R_{2}=A c R_{3}==0$

$109 \mathrm{R}_{1}=\mathrm{Ac} \mathrm{R}_{2}=\mathrm{Ac} \mathrm{R}_{3}=\beta \mathrm{OH}$

$110 R_{1}=A c R_{2}=A c R_{3}=H$

$113 \mathrm{R}_{1}=\mathrm{H} \quad \mathrm{R}_{2}=\mathrm{Ac} \quad \mathrm{R}_{3}=\beta \mathrm{OH}$

$114 \mathrm{R}_{1}=\mathrm{Ac} \quad \mathrm{R}_{2}=\mathrm{H} \quad \mathrm{R}_{3}=\beta \mathrm{OH}$

$115 \mathrm{R}_{1}=\mathrm{H} \quad \mathrm{R}_{2}=\mathrm{H} \quad \mathrm{R}_{3}=\beta \mathrm{OH}$

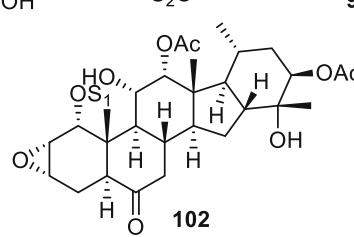

99<smiles>CC(=O)C1=CCC2CCCCC12C</smiles>

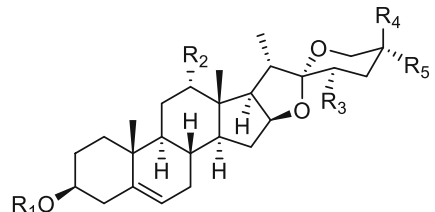

$100 \mathrm{R}_{1}=\mathrm{S}_{6} \quad \mathrm{R}_{2}=\mathrm{H} \quad \mathrm{R}_{3}=\mathrm{H} \quad \mathrm{R}_{4}=\mathrm{Me} \quad \mathrm{R}_{5}=\mathrm{H}$ $103 R_{1}=S_{2} \quad R_{2}=O H \quad R_{3}=H \quad R_{4}=H \quad R_{5}=M e$ $104 R_{1}=S_{3} R_{2}=H \quad R_{3}=O H \quad R_{4}=O H \quad R_{5}=M e$

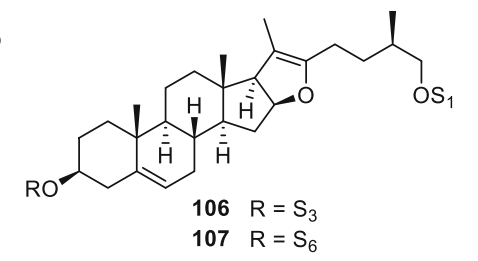

$$
\begin{aligned}
& \mathrm{S}_{1}=\beta \text {-D-GlC- } \mathrm{S}_{2}=\alpha \text {-L-Rha- }(1 \rightarrow 5)-\beta \text {-D-Api- } \quad \mathrm{S}_{3}=\alpha \text {-L-Rha- }(1 \rightarrow 2)-[\alpha-\mathrm{L}-\mathrm{Rha}-(1 \rightarrow 3)]-\beta \text {-D-GlC- } \quad \mathrm{S}_{4}=\alpha \text {-L-Rha- }(1 \rightarrow 2)-[\alpha-\mathrm{L}-\mathrm{Rha}-(1 \rightarrow 3)]-(6-\mathrm{AC})-\beta \text {-D-GIC- } \\
& \mathrm{S}_{5}=\alpha \text {-L-Rha- }(1 \rightarrow 2)-[\alpha-\mathrm{L}-\mathrm{Rha}-(1 \rightarrow 4)]-(6-\mathrm{AC})-\beta \text {-D-GlC- } \quad \mathrm{S}_{6}=\alpha \text {-L-Rha- }(1 \rightarrow 2)-[\beta \text {-D-GlC }(1 \rightarrow 3)-\mathrm{L}-\mathrm{Rha}-(1 \rightarrow 3)]-\beta \text {-D-GlC- }
\end{aligned}
$$

Fig. 4 New steroidal sapogenins and saponins from Tacca species 
Table 4 New steroidal sapogenins and saponins from Tacca species

\begin{tabular}{|c|c|c|c|c|}
\hline Nos. & Names & Species & Parts & References \\
\hline 92 & Taccaoside E & T. plantaginea & Whole plants & {$[53]$} \\
\hline 93 & Taccaoside F & T. plantaginea & Whole plants & {$[53]$} \\
\hline 94 & Taccaoside G & T. plantaginea & Whole plants & {$[53]$} \\
\hline 95 & Taccaoside $\mathrm{H}$ & T. plantaginea & Whole plants & {$[53]$} \\
\hline 96 & Taccaoside I & T. plantaginea & Whole plants & {$[53]$} \\
\hline 97 & Taccaoside $\mathbf{J}$ & T. plantaginea & Whole plants & {$[53]$} \\
\hline 98 & Taccaoside $\mathrm{K}$ & T. plantaginea & Whole plants & {$[53]$} \\
\hline 99 & Taccaoside L & T. plantaginea & Whole plants & {$[53]$} \\
\hline 100 & Taccaoside C & T. plantaginea & Whole plants & {$[54]$} \\
\hline 101 & Taccaoside D & T. plantaginea & Whole plants & {$[54]$} \\
\hline 102 & Taccasuboside A & T. subflabellata & Whole plants & {$[55]$} \\
\hline 103 & Taccasuboside B & T. subflabellata & Whole plants & {$[55]$} \\
\hline 104 & Taccasuboside C & T. subflabellata & Whole plants & {$[55]$} \\
\hline 105 & Taccasuboside D & T. subflabellata & Whole plants & {$[55]$} \\
\hline 106 & Taccaoside A & T. plantaginea & Rhizomes & {$[56]$} \\
\hline 107 & Taccaoside B & T. plantaginea & Rhizomes & {$[56]$} \\
\hline 108 & Plantagiolide A & T. plantaginea & Whole plants & {$[57]$} \\
\hline 109 & Plantagiolide B & T. plantaginea & Whole plants & {$[57]$} \\
\hline 110 & Plantagiolide $\mathrm{C}$ & T. plantaginea & Whole plants & {$[57]$} \\
\hline 111 & Plantagiolide D & T. plantaginea & Whole plants & {$[57]$} \\
\hline 112 & Plantagiolide E & T. plantaginea & Whole plants & {$[57]$} \\
\hline 113 & Plantagiolide F & T. plantaginea & Whole plants & {$[58]$} \\
\hline 114 & Plantagiolide K & T. plantaginea & Whole plants & [59] \\
\hline 115 & Plantagiolide L & T. plantaginea & Whole plants & [59] \\
\hline 116 & Plantagiolide M & T. plantaginea & Whole plants & [59] \\
\hline 117 & Plantagiolide $\mathrm{N}$ & T. plantaginea & Whole plants & [59] \\
\hline 118 & Taccalonolide W & T. plantaginea & Whole plants & {$[60]$} \\
\hline 119 & Taccalonolide X & T. plantaginea & Whole plants & {$[60]$} \\
\hline 120 & Taccalonolide Y & T. plantaginea & Whole plants & {$[60]$} \\
\hline
\end{tabular}

pennogenin-type saponins, paris saponin VI (135), pennogenin 3-O- $\beta$-chacotrioside (138), and paris saponin VII (141) were obtained and further proved to display maximal induced platelet aggregation rates (MPARs) of 72, 71, and $62 \%$ with $\mathrm{EC}_{50}$ values of $0.49,0.20$, and $0.11 \mathrm{mM}$, respectively [51]. The results also suggested that the hydroxy group at C-17 in pennogenin saponins was indispensable for their hemostatic effects, whereas the introduction of different functional groups in the A, B, or F-ring of pennogenin glycosides could make the hemostatic effect weak or disappear. Interestingly, the total saponin moieties from the above-ground parts and the rhizomes of PPY showed equivalent maximal platelet aggregation rates of 45 and $43 \%$ at a concentration of $1.5 \mathrm{mg} / \mathrm{mL}$, respectively [61]. This indicated that the above-ground parts can be an alternative and more sustainable sources for RP. Additionally, two diosgenin-type saponins, ypsilandroside $\mathrm{M}$ (49), ypsiparoside C (54), and paris saponin II (133) isolated from $Y$. parviflora, exhibited MPARs of 43, 44 and
$55 \%$ at the concentration of $0.3 \mathrm{mg} / \mathrm{mL}$, respectively [41]. This indicated that the carbonyl group at C-12 or the sole $\alpha$-L-rhamnopyranosyl-( $1 \rightarrow 4)$ - $\alpha$-L-rhamnopyranosyl- $(1 \rightarrow$ 4)-[ $\alpha$-L-rhamnopyranosyl-( $1 \rightarrow 2)]$ - $\beta$-D-glucopyranosyl moiety at $\mathrm{OH}-3$ was essential for the hemostatic effect of diosgenin saponins.

\subsection{Cytotoxic Effect}

A number of saponins were proved to have cytotoxicity against various human tumor cells. Two Trillium saponis with a double bond between C-13 and 14 isolated from $T$. kamtschaticum, trillikamtosides P (89) and R (91), showed cytotoxic effect against HCT116 (colorectal carcinoma) cells with the MIC values of 4.92 and $5.84 \mu \mathrm{M}$, respectively [52]. Ypsilandroside $\mathrm{G}$ (43) obtained from $Y$. thibetica displayed cytotoxic effect against K562 (leukemia) cells with an MIC value of $4.7 \mu \mathrm{M}$, and paris saponin VII (141) identified from the same species was cytotoxic 


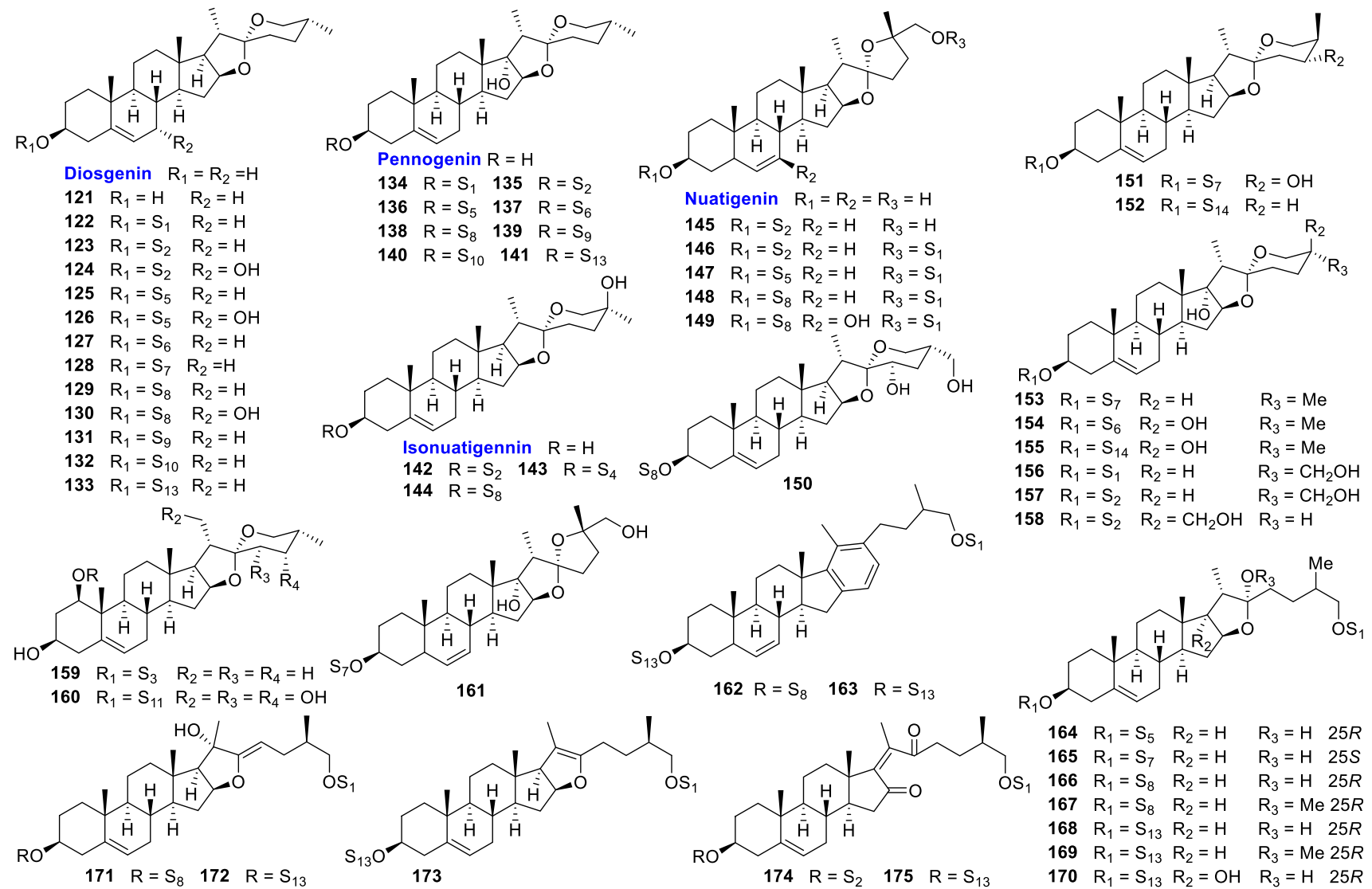

Fig. 5 Known steroidal sapogenins and saponins

towards SPC-A-1 (lung carcinoma) and BGC-823 (gastric carcinoma) with the $\mathrm{IC}_{50}$ values of 2.6 and $4.0 \mu \mathrm{M}$, respectively [38]. Nuatigenin 3-O- $\alpha$-L-rhamnopyranosyl$(1 \rightarrow 2)-\beta$-D-glucopyranoside isolated from the stems and leaves of PPY exhibited cytotoxicity against HepG2 (hepatoma) and HEK293 (renal carcinoma) cell lines with $\mathrm{IC}_{50}$ values of 2.9 and $5.0 \mu \mathrm{M}$, respectively [27]. Taccaoside (128), a saponin obtained from $T$. plantaginea, exhibited significant cytotoxicity against HepG2 and HEK293 cell lines with $\mathrm{IC}_{50}$ values of 1.2 and $1.7 \mu \mathrm{M}$, respectively [53]. Compared with the positive control drug cisplatin (DDP), a furostanol saponin isolated from $T$.

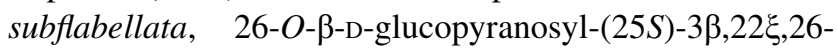
triol-furost-5-ene 3 - $O$ - $\alpha$-L-rhamnopyranosyl $(1 \rightarrow 2)$ - $[\alpha-\mathrm{L}-$ rhamnopyranosyl $(1 \rightarrow 3)]-\beta$-D-glucopyranoside

(165) showed significant cytotoxicity against HL-60 (leukemic), SMMC-7721 (hepatoma), A549 (lung carcinoma), MCF-7 (breast carcinoma), and SW480 (colon carcinoma) cells with the $\mathrm{IC}_{50}$ values of $4.63,4.34,3.00,11.13$, and $2.68 \mu \mathrm{M}$, respectively [55]. Ypsilandroside P (62), a furostanol saponin obtained from $Y$. thibetica, showed inhibition ratio of 86.4 and $75.9 \%$ to A549 and HL-60 cells at the concentration of $10.0 \mu \mathrm{M}$, respectively [43]. Moreover, the total saponin moieties from the both rhizomes and above-ground parts of PPY showed cytotoxicities against HL-60, A549, SMMC-7721, MCF-7, and SW480 cells [61]. To be more specific, the former displayed cytotoxicities against above-mentioned cancer cells with $\mathrm{IC}_{50}$ values of $1.77,1.75,5.23,6.62$, and $3.49 \mu \mathrm{M}$, whereas the latter was less cytotoxic with $\mathrm{IC}_{50}$ values of $9.54,9.30$, $12.61,8.12$, and $11.25 \mu \mathrm{M}$, respectively.

\subsection{Antimicrobial Effect}

Ypsilandroside G (43) obtained from $Y$. thibetica showed moderate inhibitory effect on Candida albicans with an MIC value of $10 \mu \mathrm{g} / \mathrm{mL}$ [38]. Compared with that of fluconazole $(\mathrm{MIC}=52.3 \mu \mathrm{M})$, five saponins isolated from $T$. kamtschaticum, named paris saponin VI (135), floribundasaponin B (136), pennogenin 3-O- $\beta$-chacotrioside (138), paris saponin V (123), and ophiopogonin B (159), displayed significant antifungal activity against $C$. albicans 


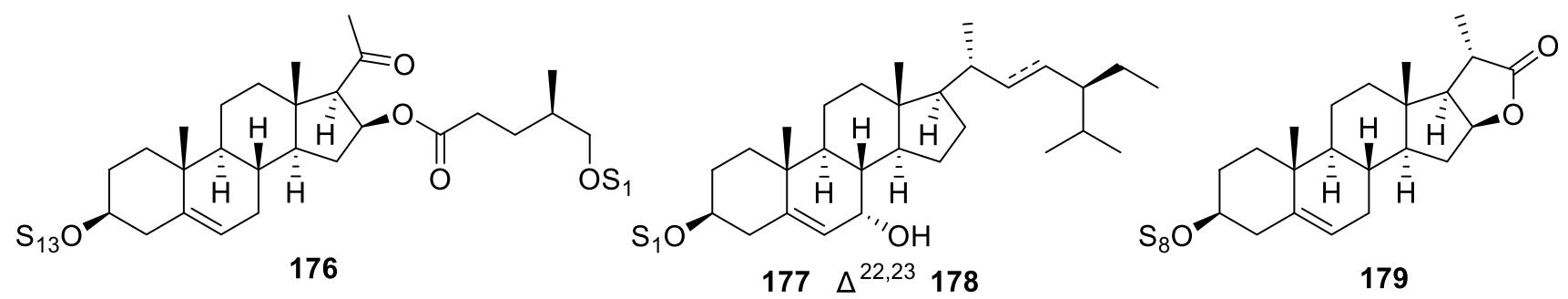

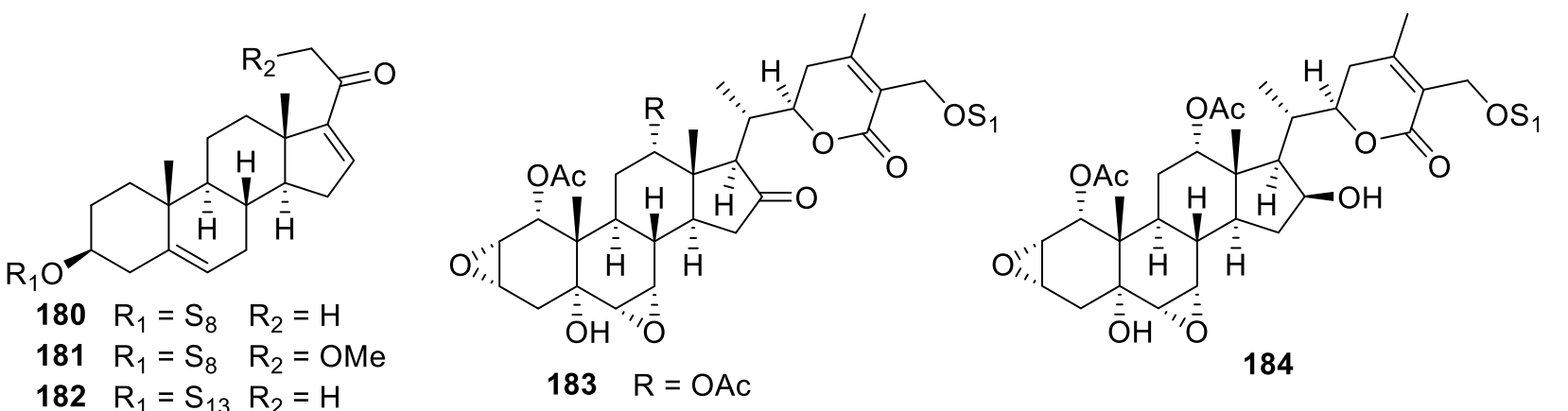

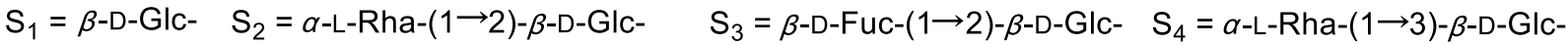

$$
\begin{aligned}
& \mathrm{S}_{5}=\alpha \text {-L-Rha- }(1 \rightarrow 4)-\beta \text {-D-Glc- } \quad \mathrm{S}_{6}=\alpha \text {-L-Rha- }(1 \rightarrow 2)-[\beta \text {-D-GlC- }(1 \rightarrow 3)]-\beta \text {-D-Glc- } \\
& \mathrm{S}_{7}=\alpha \text {-L-Rha- }(1 \rightarrow 2)-[\alpha-\mathrm{L}-\mathrm{Rha}-(1 \rightarrow 3)]-\beta \text {-D-GlC- } \quad \mathrm{S}_{8}=\alpha \text {-L-Rha- }(1 \rightarrow 2)-[\alpha-\mathrm{L}-\mathrm{Rha}-(1 \rightarrow 4)]-\beta \text {-D-GlC- }
\end{aligned}
$$

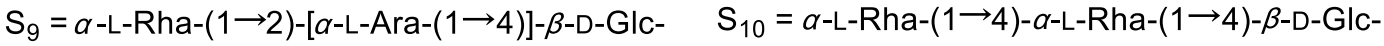

$$
\begin{aligned}
& \mathrm{S}_{11}=\beta \text {-D-Api- }(1 \rightarrow 3)-\alpha \text {-L-Rha- }(1 \rightarrow 2)-[\beta \text {-D-Xyl- }(1 \rightarrow 3)]-\alpha \text {-L-Ara- } \\
& \mathrm{S}_{12}=\alpha \text {-L-Rha- }(1 \rightarrow 2)-[\alpha-\mathrm{L}-\mathrm{Rha}-(1 \rightarrow 4)-\alpha \text {-L-Rha- }(1 \rightarrow 3)]-\beta \text {-D-GlC- } \\
& \mathrm{S}_{13}=\alpha \text {-L-Rha- }(1 \rightarrow 2)-[\alpha \text {-L-Rha- }(1 \rightarrow 4)-\alpha \text {-L-Rha- }(1 \rightarrow 4)]-\beta \text {-D-GlC- } \\
& \mathrm{S}_{14}=\alpha \text {-L-Rha- }(1 \rightarrow 2)-[\beta \text {-D-GlC- }(1 \rightarrow 4)-\alpha-\mathrm{L}-\mathrm{Rha}-(1 \rightarrow 4)]-\beta \text {-D-GlC- }
\end{aligned}
$$

Fig. 5 continued

with the MIC values of 21.1, 10.6, 8.8, 21.6, and $11.0 \mu \mathrm{M}$, respectively [51]. Chonglouoside SL-6 (6), progenin II (125), and dumoside (179), three steroidal saponins isolated from the stems and leaves, exhibited good antibacterial activity with the MIC values of 3.9, 7.8, and $3.9 \mu \mathrm{g} /$ $\mathrm{mL}$, respectively [25, 26]. All three spirostanol saponins identified from PPY, paris saponin V (123), dioscin (129), and paris saponin II (133), were revealed to show significant antifungal activities against $C$. albicans 5314 and $C$. albicans Y0109 with an MIC value of $1.95 \mu \mathrm{g} / \mathrm{mL}$ [61]. Also, the total saponin moieties from both the aboveground parts and the rhizomes of PPY exhibited remarkable antifungal activities against $C$. albicans Y0109 with MIC values of 10.3 and $5.15 \mu \mathrm{g} / \mathrm{mL}$, respectively, compared with that the positive control voriconazole $(\mathrm{MIC}=$ $15.63 \mu \mathrm{g} / \mathrm{mL}$ ) [61].

\section{Conclusion}

In summary, our continuous effort to search for alternative resources of RP led to the isolation of 184 steroidal derivatives, including 120 new ones. More importantly, several compounds of them displayed remarkable hemostatic, cytotoxic, and antimicrobial effects. Our studies disclosed that the non-medicinal parts of PPY, as well as other plants of Paris, Ypsilandra, Trillium, and Taccaceae family are also resources rich of steroidal saponins similar to those of RP, especially those recorded in Chinese Pharmacopoeia, namely, paris saponins I (131), II (133), VI (135), and VII (141). However, the investigations on the total content of these saponins, the related bioactivities of total saponin moieties of the studied species compared with those of RP, and their security capability are quite indispensable to confirm that whether the non-medicinal parts of PPY and other species from Paris, Ypsilandra, and Tacca genera could be safe and dependable alternative resources of RP. The arial parts of PPY and the whole plants of $T$. kamtschaticum might be alternative resources for RP based on the fact that they shared the same or similar saponins and bioactivities. The continuous studies on the saponin constituents of non-medicinal parts of RP and other plants will be carried out in our laboratory which may led to the discovery of more alternative resources for RP. 
Table 5 Known steroidal sapogenins and saponins

\begin{tabular}{|c|c|c|c|c|}
\hline Nos. & Names & Species & Parts & References \\
\hline 121 & Diosgenin & PPY & Stems and leaves & {$[25]$} \\
\hline 122 & Polyphyllin A & PPY & Stems and leaves & {$[25]$} \\
\hline \multirow[t]{4}{*}{123} & \multirow[t]{4}{*}{ Paris saponin V } & PPY & Stems and leaves & {$[25]$} \\
\hline & & P. axialis & rhizomes & {$[31]$} \\
\hline & & P. delavayi & Rhizomes & {$[31]$} \\
\hline & & Y. thibetica & Whole plants & {$[38]$} \\
\hline 124 & Sansevierin A & PPY & Stems and leaves & {$[25]$} \\
\hline 125 & Progenin II & PPY & Stems and leaves & {$[25]$} \\
\hline 126 & Disoseptemloside D & PPY & Stems and leaves & {$[25]$} \\
\hline 127 & $\begin{array}{l}\text { Diosgenin-3-O- } \beta \text {-D-glucopyranosyl- }(1 \rightarrow 3) \text { - } \\
\quad[\alpha \text {-L-rhamnopyranosyl }(1 \rightarrow 2)]-\beta \text {-D-glucopyranoside }\end{array}$ & P. axialis & Rhizomes & {$[30]$} \\
\hline \multirow[t]{2}{*}{128} & \multirow[t]{2}{*}{ Taccaoside } & T. plantaginea & Whole plants & {$[53]$} \\
\hline & & T. chanteraeri & Rhizomes & {$[66]$} \\
\hline \multirow[t]{3}{*}{129} & \multirow[t]{3}{*}{ Dioscin } & PPY & Stems and leaves & {$[25]$} \\
\hline & & Y. thibetica & Whole plants & {$[38]$} \\
\hline & & T. plantaginea & Whole plants & {$[53]$} \\
\hline 130 & Disoseptemloside E & PPY & Stems and leaves & {$[25]$} \\
\hline \multirow[t]{4}{*}{131} & \multirow[t]{4}{*}{ Paris saponin I } & P. axialis & Rhizomes & {$[31]$} \\
\hline & & P. delavayi & Rhizomes & {$[31]$} \\
\hline & & P. dunniana & Rhizomes & {$[31]$} \\
\hline & & P. luquanensis & Rhizomes & {$[62]$} \\
\hline \multirow[t]{3}{*}{132} & \multirow{3}{*}{$\begin{array}{l}\text { Diosgenin-3- } O \text { - } \alpha \text {-L-rhamnopyranosyl- }(1 \rightarrow 4) \text { - } \\
\alpha \text {-L-rhamnopyranosyl }(1 \rightarrow 4)-\beta \text {-D-glucopyranoside }\end{array}$} & PPY & Stems and leaves & {$[27]$} \\
\hline & & P. verticillata & Aerial parts & {$[32]$} \\
\hline & & Y. thibetica & Whole plants & {$[38]$} \\
\hline \multirow[t]{3}{*}{133} & \multirow[t]{3}{*}{ Paris saponin II } & PPY & Stems and leaves & {$[25]$} \\
\hline & & Y. thibetica & Whole plants & {$[38]$} \\
\hline & & $Y$. parviflora & Whole plants & {$[41]$} \\
\hline 134 & Pennogenin 3-O- $\beta$-D-glucopyranoside & T. kamtschaticum & Whole plants & {$[51]$} \\
\hline \multirow[t]{4}{*}{135} & \multirow[t]{4}{*}{ Paris saponin VI } & PPY & Stems and leaves & {$[25]$} \\
\hline & & P. axialis & Rhizomes & {$[31]$} \\
\hline & & P. delavayi & Rhizomes & {$[31]$} \\
\hline & & T. kamtschaticum & Whole plants & {$[51]$} \\
\hline \multirow[t]{2}{*}{136} & \multirow[t]{2}{*}{ Floribundasaponin B } & Y. thibetica & Whole plants & {$[38]$} \\
\hline & & T. kamtschaticum & Whole plants & {$[51]$} \\
\hline 137 & $\begin{array}{l}\text { Pennogenin } 3 \text { - } O \text { - } \beta \text {-D-glucopyranosyl- }(1 \rightarrow 3) \text { - } \\
\quad[\alpha \text {-L-rhamnopyranosyl }(1 \rightarrow 2)] \text { - } \beta \text {-D-glucopyranoside }\end{array}$ & P. axialis & Rhizomes & {$[31]$} \\
\hline \multirow[t]{4}{*}{138} & \multirow[t]{4}{*}{ Pennogenin $3-O-\beta$-chacotrioside } & PPY & Aerial parts & {$[64]$} \\
\hline & & T. kamtschaticum & Whole plants & {$[51]$} \\
\hline & & Y. thibetica & Whole plants & {$[38]$} \\
\hline & & Y. parviflora & Whole plants & {$[41]$} \\
\hline \multirow[t]{4}{*}{139} & \multirow[t]{4}{*}{ Paris saponin $\mathrm{H}$} & P. axialis & Rhizomes & {$[31]$} \\
\hline & & P. delavayi & Rhizomes & {$[31]$} \\
\hline & & P. dunniana & Rhizomes & {$[31]$} \\
\hline & & P. luquanensis & Rhizomes & {$[62]$} \\
\hline \multirow[t]{4}{*}{140} & \multirow{4}{*}{$\begin{array}{l}\text { Pennogenin } 3-O-\alpha \text {-L-rhamnopyranosyl-( }(1 \rightarrow 4) \text { - } \\
\alpha \text {-L-rhamnopyranosyl-( }(1 \rightarrow 4) \text { - } \beta \text {-D-glucopyranoside }\end{array}$} & PPY & Stems and leaves & {$[25]$} \\
\hline & & P. verticillata & Aerial parts & {$[32]$} \\
\hline & & T. kamtschaticum & Whole plants & {$[51]$} \\
\hline & & Y. parviflora & Whole plants & {$[48]$} \\
\hline
\end{tabular}


Table 5 continued

\begin{tabular}{|c|c|c|c|c|}
\hline Nos. & Names & Species & Parts & References \\
\hline \multirow[t]{7}{*}{141} & \multirow[t]{7}{*}{ Paris saponin VII } & PPY & Stems and leaves & {$[25]$} \\
\hline & & P. verticillata & Aerial parts & {$[32]$} \\
\hline & & P. luquanensis & Rhizomes & {$[62]$} \\
\hline & & PPY & Seeds & {$[63]$} \\
\hline & & T. kamtschaticum & Whole plants & {$[51]$} \\
\hline & & Y. parviflora & Whole plants & [41] \\
\hline & & Y. thibetica & Whole plants & [39] \\
\hline 142 & $\begin{array}{l}\text { Isonuatigenin 3-O- } \alpha \text {-L-rhamnopyranosyl- } \\
\quad(1-2)-\beta \text {-D-glucopyranoside }\end{array}$ & PPY & Stems and leaves & {$[25]$} \\
\hline 143 & Disoseptemloside H & PPY & Stems and leaves & {$[25]$} \\
\hline 144 & $\begin{array}{l}\text { Pennogenin } 3 \text { - } O \text { - } \alpha \text {-L-rhamnopyranosyl- }(1 \rightarrow 2) \text { - } \\
\quad[\alpha \text {-L-rhamnopyranosyl }(1 \rightarrow 4)]-\beta \text {-D-glucopyranoside }\end{array}$ & PPY & Aerial parts & {$[65]$} \\
\hline 145 & Nuatigenin 3-O- $\alpha$-L-rhamnopyranosyl-( $(1 \rightarrow 2)$ - $\beta$-D-glucopyranoside & PPY & Stems and leaves & {$[27]$} \\
\hline 146 & $\begin{array}{l}\text { 26- } O \text { - } \beta \text {-D-glucopyranosyl nuatigenin } 3-O-\alpha \text {-L-rhamnopyranosyl- } \\
(1 \rightarrow 2)-\beta \text {-D-glucopyranoside }\end{array}$ & PPY & Stems and leaves & {$[27]$} \\
\hline 147 & $\begin{array}{l}\text { 26- } O \text { - } \beta \text {-D-glucopyranosyl nuatigenin 3- } O \text { - } \alpha \text {-L-rhamnopyranosyl- } \\
(1 \rightarrow 4)-\beta \text {-D-glucopyranoside }\end{array}$ & PPY & Stems and leaves & {$[27]$} \\
\hline 148 & $\begin{array}{l}\text { 26- } O \text { - } \beta \text {-D-glucopyranosyl nuatigenin } 3-O-\alpha-\mathrm{L} \text {-rhamnopyranosyl- } \\
(1 \rightarrow 2) \text {-[ } \alpha-\mathrm{L} \text {-rhamnopyranosyl-( }(1 \rightarrow 4)]-\beta \text {-D-glucopyranoside }\end{array}$ & PPY & Aerial parts & {$[65]$} \\
\hline 149 & Abutiloside L & PPY & Stems and leaves & {$[27]$} \\
\hline 150 & Borassoside B & PPY & Stems and leaves & {$[27]$} \\
\hline 151 & $\begin{array}{l}(24 S, 25 R) \text {-spirost-5-en-3 } \beta, 24 \text {-diol-3- } O \text { - } \alpha \text {-L-rhamnopyranosyl- } \\
\quad(1 \rightarrow 2) \text {-[ } \alpha \text {-L-rhamnopyranosyl }(1 \rightarrow 3)]-\beta \text {-D-glucopyranoside }\end{array}$ & T. plantaginea & Whole plants & {$[53]$} \\
\hline 152 & 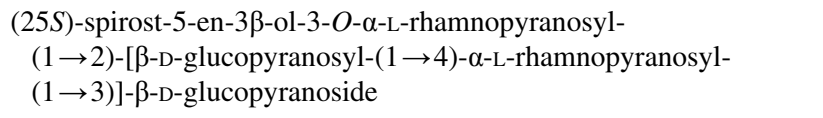 & T. plantaginea & Whole plants & {$[53]$} \\
\hline 153 & Spiroconazole A & T. plantaginea & Whole plants & {$[53]$} \\
\hline 154 & Diosbulbiside A & T. plantaginea & Whole plants & {$[53]$} \\
\hline 155 & Diosbulbiside B & T. plantaginea & Whole plants & {$[53]$} \\
\hline 156 & (25S)-27-hydroxypennogenin 3-O- $\beta$-D-glucopyranoside & T. kamtschaticum & Whole plants & {$[51]$} \\
\hline 157 & $\begin{array}{l}\text { (25S)-27-hydroxypennogenin-3- } O \text { - } \alpha \text {-L-rhamnopyranosyl- } \\
(1 \rightarrow 2) \text { - } \beta \text {-D-glucopyranoside }\end{array}$ & T. kamtschaticum & Whole plants & {$[51]$} \\
\hline 158 & Trikamsteroside A & T. kamtschaticum & Whole plants & {$[51]$} \\
\hline 159 & Ophiopogonin B & T. kamtschaticum & Whole plants & {$[51]$} \\
\hline 160 & Trikamsteroside E & T. kamtschaticum & Whole plants & {$[52]$} \\
\hline 161 & Diosbulbiside E & T. plantaginea & Whole plants & {$[53]$} \\
\hline 162 & Aethioside A & T. kamtschaticum & Whole plants & {$[52]$} \\
\hline \multirow[t]{3}{*}{163} & \multirow[t]{3}{*}{ Parispseudoside A } & P. verticillata & Aerial parts & {$[32]$} \\
\hline & & Y. parviflora & Whole plants & {$[41]$} \\
\hline & & Y. thibetica & Whole plants & {$[43]$} \\
\hline 164 & Protoprogenin II & Y. thibetica & Whole plants & {$[43]$} \\
\hline \multirow[t]{2}{*}{165} & \multirow{2}{*}{$\begin{array}{l}\text { 26- } O \text { - } \beta \text {-D-glucopyranosyl- }(25 S)-3 \beta, 22 \xi, 26 \text {-triol- } \\
\text { furost-5-ene 3- } O \text { - } \alpha \text {-L-rhamnopyranosyl- }(1 \rightarrow 2) \\
\text {-[ } \alpha \text {-L-rhamnopyranosyl }(1 \rightarrow 3)]-\beta \text {-D-glucopyranoside }\end{array}$} & T. plantaginea & Whole plants & {$[54]$} \\
\hline & & T. subflabellata & Whole plants & {$[55]$} \\
\hline 166 & Proto-dioscin & PPY & Stems and leaves & {$[25]$} \\
\hline 167 & Methylprotodioscin & PPY & Stems and leaves & {$[25]$} \\
\hline \multirow[t]{3}{*}{168} & \multirow[t]{3}{*}{ Proto-paris saponin II } & P. verticillata & Aerial parts & {$[32]$} \\
\hline & & Y. parviflora & Whole plants & {$[41]$} \\
\hline & & Y. thibetica & Whole plants & {$[43]$} \\
\hline
\end{tabular}


Table 5 continued

\begin{tabular}{|c|c|c|c|c|}
\hline Nos. & Names & Species & Parts & References \\
\hline 169 & 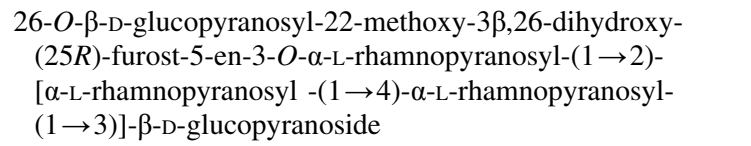 & P. verticillata & Aerial parts & {$[32]$} \\
\hline \multirow[t]{2}{*}{170} & \multirow[t]{2}{*}{ Proto-paris saponin VII } & P. verticillata & Aerial parts & {$[32]$} \\
\hline & & Y. thibetica & Whole plants & {$[43]$} \\
\hline 171 & $\begin{array}{l}\text { 26- } O \text { - } \beta \text {-D-glucopyranosyl-3 } \beta, 20 \alpha, 26 \text {-triol- }(25 R) \text { - } \\
\text { 5,22-dienofurostan } 3-O \text { - } \alpha \text {-L-rhamnopyranosyl- } \\
(1 \rightarrow 2) \text {-[ } \alpha \text {-L-rhamnopyranosyl }(1 \rightarrow 4)]-\beta \text {-D-glucopyranoside }\end{array}$ & PPY & Stems and leaves & {$[25]$} \\
\hline \multirow[t]{2}{*}{172} & \multirow[t]{2}{*}{ Smilaxchinoside B } & P. verticillata & Aerial parts & {$[32]$} \\
\hline & & Y. thibetica & Whole plants & {$[43]$} \\
\hline 173 & $\begin{array}{l}\text { 26- } O \text { - } \beta \text {-D-glucopyranosyl-17(20)-dehydrokryptogenin- } \\
\text { 3- } O \text { - } \alpha \text {-L-rhamnopyranosyl- }(1 \rightarrow 2) \text { - } \beta \text {-D-glucopyranoside }\end{array}$ & T. kamtschaticum & Whole plants & {$[52]$} \\
\hline \multirow[t]{3}{*}{174} & \multirow[t]{3}{*}{ Pseudoproto $\mathrm{Pb}$} & P. verticillata & Aerial parts & {$[32]$} \\
\hline & & Y. parviflora & Whole plants & {$[41]$} \\
\hline & & Y. thibetica & Whole plants & {$[43]$} \\
\hline \multirow[t]{3}{*}{175} & \multirow[t]{3}{*}{ Parispseudoside C } & P. verticillata & Aerial parts & {$[32]$} \\
\hline & & Y. thibetica & Whole plants & {$[43]$} \\
\hline & & Y. yunnanensis & Whole plants & {$[45]$} \\
\hline 176 & 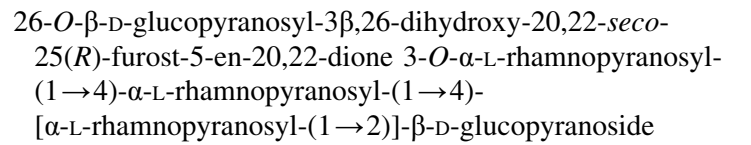 & Y. thibetica & Whole plants & {$[43]$} \\
\hline 177 & $7 \alpha$-Hydroxystigmasterol-3- $O-\beta$-D-glucopyranoside & PPY & Stems and leaves & {$[27]$} \\
\hline 178 & $7 \alpha$-Hydroxysitosterol-3- $O-\beta$-D-glucopyranoside & PPY & Stems and leaves & [27] \\
\hline 179 & Dumoside & PPY & Stems and leaves & [26] \\
\hline \multirow[t]{2}{*}{180} & \multirow[t]{2}{*}{ Hypoglaucin $\mathrm{H}$} & PPY & Stems and leaves & {$[25]$} \\
\hline & & PPY & Aerial parts & {$[64]$} \\
\hline 181 & $\begin{array}{l}\text { 21-Methoxyl pregna-5,16-dien-3 } \beta \text {-ol-20-one } 3-O-\alpha \text {-L- } \\
\text { rhamnopyranosyl- }(1 \rightarrow 2) \text {-[ } \alpha \text {-L-rhamnopyranosyl } \\
(1 \rightarrow 4)]-\beta \text {-D-glucopyranoside }\end{array}$ & PPY & Stems and leaves & {$[25]$} \\
\hline \multirow[t]{3}{*}{182} & \multirow{3}{*}{$\begin{array}{l}\text { Pregna-5,16-dien-3 } \beta \text {-ol-20-one } 3-O \text { - } \alpha \text {-L-rhamnopyranosyl- } \\
\quad(1 \rightarrow 2) \text {-[ } \alpha \text {-L-rhamnopyranosyl }(1 \rightarrow 4) \text { - } \alpha \text {-L-rhamnopyranosyl } \\
(1 \rightarrow 4)] \text { - } \beta \text {-D-glucopyranoside }\end{array}$} & P. verticillata & Aerial parts & {$[32]$} \\
\hline & & Y. thibetica & Whole plants & {$[43]$} \\
\hline & & PPY & Aerial parts & {$[64]$} \\
\hline \multirow[t]{2}{*}{183} & \multirow[t]{2}{*}{ Chantriolide A } & T. subflabellata & Whole plants & {$[55]$} \\
\hline & & T. plantaginea & Whole plants & {$[57]$} \\
\hline 184 & Chantriolide B & T. subflabellata & Whole plants & {$[55]$} \\
\hline
\end{tabular}

Acknowledgements This work was financially supported by the National Natural Science Foundation of China (Nos. 31570363, 31770391, and 31600283), the Natural Science Foundation of Yunnan Province (2015FA031 and 2017FB128), the Science and Technology Research Program (Grant No. KIB2016001) of Kunming Institute of Botany, CAS, Guiding Program of Interdisciplinary Studies from Kunming Institute of Botany, CAS (Grant No. KIB2017004), and the Foundation of State Key Laboratory of Phytochemistry and Plant Resources in West China (P2017-ZZ04), Kunming Institute of Botany, Chinese Academy of Sciences.

\section{Compliance with Ethical Standards}

Conflict of interest All authors declare no conflict of interest.
Open Access This article is distributed under the terms of the Creative Commons Attribution 4.0 International License (http://creative commons.org/licenses/by/4.0/), which permits unrestricted use, distribution, and reproduction in any medium, provided you give appropriate credit to the original author(s) and the source, provide a link to the Creative Commons license, and indicate if changes were made.

\section{References}

1. H. Li, The Genus Paris (Trilliaceae) (Science Press, Beijing, 1998), pp. 12-16

2. Y.H. Ji, P.W. Fritsch, H. Li, T.J. Xiao, Z.K. Zhou, Ann. Bot. 98 245-256 (2006) 
3. H. Li, L.G. Lei, Y.M. Yang, J. West China For. Sci. 46, 1-5 (2017)

4. Y.H. Ji, C.J. Yang, Y.L. Huang, Phytotaxa 306, 234-236 (2017)

5. Z. Wang, X.Z. Cai, Z.X. Zhong, Z. Xu, N. Wei, J.F. Xie, G.W. Hu, Q.F. Wang, Phytotaxa 314, 145-149 (2017)

6. Y.Y. Liu, D.D. Luo, H. Yao, X.C. Zhang, L.W. Yang, B.Z. Duan, Phytotaxa 326, 297-300 (2017)

7. J. Yang, Y.H. Wang, H. Li, Phytotaxa 329, 193-196 (2017)

8. Chinese Pharmacopoeia Commission, Pharmacopoeia of the People's Republic of China, vol. 1 (China Medical Science Press, Beijing, 2010), pp. 243-244

9. T. Nohara, H. Yabuta, M. Suenobu, R. Hida, K. Miyahara, T. Kawasaki, Chem. Pharm. Bull. 21, 1240-1247 (1973)

10. S.B. Singh, R.S. Thakur, H.R. Schulten, Phytochemistry 21, 2925-2929 (1980)

11. C.X. Chen, Y.T. Zhang, J. Zhou, Acta Bot. Yunnanica 5, 91-97 (1983)

12. J.Y. Zhang, Y.Z. Wang, Y.L. Zhao, S.B. Yang, Z.T. Zuo, M.Q. Yang, J. Zhang, W.Z. Yang, T.-M. Yang, H. Jin, J. Asian Nat. Prod. Res. 13, 670-681 (2011)

13. Y. Mimaki, M. Kuroda, Y. Obata, Y. Sashida, M. Kitahara, A. Yasuda, N. Naoi, Z.W. Xu, M.R. Li, A.N. Lao, Nat. Prod. Lett. 14, 357-364 (2000)

14. Y. Zhao, L.P. Kang, Y.X. Liu, Y.G. Liang, D.W. Tan, Z.Y. Yu, Y.W. Cong, B.P. Ma, Planta Med. 75, 356-363 (2009)

15. X. Wu, L. Wang, H. Wang, Y. Dai, W.C. Ye, Y.L. Li, Phytochemistry 81, 133-143 (2012)

16. L.P. Kang, Y.X. Liu, T. Eichhorn, E. Dapat, H.S. Yu, Y. Zhao, C. Q. Xiong, C. Liu, T. Efferth, B.P. Ma, J. Nat. Prod. 75, 12011205 (2012)

17. Y.S. Chen, Y. He, C. Chen, Y. Zeng, D. Xue, F.Y. Wen, L. Wang, H. Zhang, J.R. Du, Steroids 83, 39-44 (2014)

18. F.Y. Long, Y.S. Chen, L. Zhang, X. Kuang, Y. Yu, L.F. Wang, X. J. Liu, L. Wang, Y.F. Zhou, N. Sang, J.R. Du, J. Ethnopharmacol. 162, 112-120 (2015)

19. Y.S. Wen, W. Ni, X.J. Qin, H. Yan, C.X. Chen, Y. Hua, Y.C. Cheng, L. He, H.Y. Liu, Phytochem. Lett. 12, 31-34 (2015)

20. S.S. Jing, Y. Wang, X. Li, S.L. Man, W.Y. Gao, Nat. Prod. Res. 31, 660-666 (2017)

21. D.W. Deng, D.R. Lauren, J.M. Cooney, D.J. Jensen, K.V. Wurms, J.E. Upritchard, R.D. Cannon, M.Z. Wang, M.Z. Li, Planta Med. 74, 1397-1402 (2008)

22. L.C. Zhu, J. Tan, B.C. Wang, L.H. Guan, Y.P. Liu, C. Zheng, Iran. J. Pharm. Res. 10, 279-286 (2011)

23. J.C.N. Ma, F.W. Lau, Phytochemistry 24, 1561-1565 (1985)

24. Y.L. Fu, Z.Y. Yu, X.M. Tang, Y. Zhao, X.L. Yuan, S. Wang, B.P. Ma, Y.W. Cong, J. Thromb. Haemost. 6, 524-533 (2008)

25. X.J. Qin, D.J. Soun, W. Ni, C.X. Chen, Y. Hua, L. He, H.Y. Liu, Steroids 77, 1242-1248 (2012)

26. X.J. Qin, C.X. Chen, W. Ni, H. Yan, H.Y. Liu, Fitoterapia 84, 248-251 (2013)

27. X.J. Qin, M.Y. Yu, W. Ni, H. Yan, C.X. Chen, Y.C. Cheng, L. He, H.Y. Liu, Phytochemistry 121, 20-29 (2016)

28. C.X. Chen, J. Zhou, H. Nagasawa, A. Suzuki, Acta Bot. Yunnanica 17, 215-220 (1995)

29. C.X. Chen, J. Zhou, Acta Bot. Yunnanica 14, 111-113 (1992)

30. C.X. Chen, J. Zhou, Acta Bot. Yunnanica 6, 111-117 (1984)

31. C.X. Chen, J. Zhou, Acta Bot. Yunnanica 9, 239-245 (1987)

32. C.L. Sun, W. Ni, H. Yan, Z.H. Liu, L. Yang, Y.A. Si, Y. Hua, C. X. Chen, L. He, J.H. Zhao, H.Y. Liu, Steroids 92, 90-95 (2014)

33. L.Y. Jin, T.X. Lu, X.J. Qin, W. Ni, H. Yan, Y. Chen, H. Liu, H.P. He, H.Y. Liu, Nat. Prod. Bioprospect. 6, 205-210 (2016)

34. K.Z. Hou, Dictionary of the Families and Genera of Chinese Seed Plants, 2nd edn. (Science Press, Beijing, 1982), pp. 521-522

35. Jiangsu New Medicinal College, Dictionary of Traditional Chinese Materia Medica (Shanghai Scientific and Technological Press, Shanghai, 1977), p. 1841
36. Yunnan Food and Drug Administration, The Yunnan Chinese Materia Medica Standards ((Yi Nationality Medicine (III)) (Yunnan Scientific and Technological Press, Kunming, 2010), pp. 5-6

37. B.B. Xie, H.Y. Liu, W. Ni, C.X. Chen, Y. Lü, L. Wu, Q.T. Zheng, Chem. Biodivers. 3, 1211-1218 (2006)

38. B.B. Xie, H.Y. Liu, W. Ni, C.X. Chen, Steroids 74, 950-955 (2009)

39. Y. Lu, C.X. Chen, W. Ni, Y. Hua, H.Y. Liu, Steroids 75, 982-987 (2010)

40. X.D. Zhang, C.X. Chen, J.Y. Yang, W. Ni, H.Y. Liu, Helv. Chim. Acta 95, 1087-1093 (2012)

41. T.X. Lu, T. Shu, X.J. Qin, W. Ni, Y.H. Ji, Q.R. Chen, A. Khan, Q. Zhao, H.Y. Liu, Steroids 123, 55-60 (2017)

42. B.B. Xie, C.X. Chen, Y.H. Guo, Y.Y. Li, Y.J. Liu, W. Ni, L.M. Yang, N.B. Gong, Y.T. Zheng, R.R. Wang, Y. Lü, H.Y. Liu, Planta Med. 79, 1063-1067 (2013)

43. H.Y. Liu, C.X. Chen, Y. Lu, J.Y. Yang, W. Ni, Nat. Prod. Bioprospect. 2, 11-15 (2012)

44. Y.A. Si, H. Yan, W. Ni, Z.H. Liu, T.X. Lu, C.X. Chen, H.Y. Liu, Nat. Prod. Bioprospect. 4, 315-318 (2014)

45. Y. Chen, Y.A. Si, H. Yan, X.J. Qin, C.X. Chen, H.Y. Liu, Nat. Prod. Bioprospect. 6, 173-182 (2016)

46. Y. Lu, B.B. Xie, C.X. Chen, W. Ni, Y. Hua, H.Y. Liu, Helv. Chim. Acta 94, 92-97 (2011)

47. F.C. Wang, J. Tang, X.Q. Chen, Z.Y. Zhang, L.K. Dai, S.Y. Liang, Y.C. Tang, L. Liu, K.Y. Lang, Flora of China, vol. 15 (Science Press, Beijing, 1978), pp. 97-98

48. Jiangsu New Medical College, Dictionary of Chinese Traditional Medicine (Zhong Yao Da Ci Dian) (Shanghai Science and Technology Press, Shanghai, 1986), pp. 831-975

49. M. Ono, C. Takamura, F. Sugita, C. Masuoka, H. Yoshimitsu, T. Ikeda, T. Nohara, Chem. Pharm. Bull. 55, 551-556 (2007)

50. A. Yokosuka, Y. Mimaki, Phytochemistry 69, 2724-2730 (2008)

51. Y. Chen, W. Ni, H. Yan, X.J. Qin, A. Khan, H. Liu, T. Shu, L.Y. Jin, H.Y. Liu, Phytochemistry 131, 165-173 (2016)

52. X.J. Qin, Y.A. Si, Y. Chen, H. Liu, W. Ni, H. Yan, T. Shu, Y.H Ji, H.Y. Liu, Bioorg. Med. Chem. Lett. 27, 2267-2273 (2017)

53. Z.H. Liu, H. Yan, Y.A. Si, W. Ni, Y. Chen, L. He, H.Y. Liu, Steroids 106, 86-92 (2016)

54. H.Y. Liu, W. Ni, X.J. Hao, C.X. Chen, J. Asian Nat. Prod. Res. 8, 293-298 (2006)

55. L. Li, W. Ni, X.R. Li, Y. Hua, P.L. Fang, L.M. Kong, L.L. Pan, Y. Li, C.X. Chen, H.Y. Liu, Steroids 76, 1037-1042 (2011)

56. H.Y. Liu, C.X. Chen, Chin. Chem. Lett. 13, 633-636 (2002)

57. H.Y. Liu, W. Ni, B.B. Xie, L.Y. Zhou, X. Wang, C.X. Chen, Chem. Pharm. Bull. 54, 992-995 (2006)

58. J.Y. Yang, C.X. Chen, R.H. Zhao, X.J. Hao, H.Y. Liu, Nat. Prod. Res. 25, 40-44 (2011)

59. Z.H. Liu, H. Yan, Y.A. Si, W. Ni, Y. Chen, C.X. Chen, L. He, Z. Q. Zhang, H.Y. Liu, Fitoterapia 105, 210-214 (2015)

60. J.Y. Yang, R.H. Zhao, C.X. Chen, W. Ni, F. Teng, X.J. Hao, H.Y. Liu, Helv. Chim. Acta 91, 1077-1082 (2008)

61. X.J. Qin, W. Ni, C.X. Chen, H.Y. Liu, J. Ethnopharmacol. 224, 134-139 (2018)

62. C.X. Chen, J. Zhou, Y.T. Zhang, C.K. Gao, Acta Bot. Yunnanica 5, 219-223 (1983)

63. C.X. Chen, H.B. Lian, Y.C. Li, J. Zhou, Acta Bot. Yunnanica 12, $452(1990)$

64. C.X. Chen, J. Zhou, Y.T. Zhang, Y.Y. Zhao, Acta Bot. Yunnanica 12, 323-329 (1990)

65. C.X. Chen, Y.T. Zhang, J. Zhou, Acta Bot. Yunnanica 17, 473478 (1995)

66. J. Zhou, C.X. Chen, R.M. Liu, C.R. Yang, Acta Bot. Sin. 25, 568-573 (1983) 